OPEN ACCESS

Edited by:

Isabela Ramos,

Federal University of Rio de Janeiro,

Brazi

Reviewed by:

Sheila Ons,

National University of La Plata,

Argentina

Gerd Gäde

University of Cape Town, South Africa

David Majerowicz,

Federal University of Rio de Janeiro,

Brazil

${ }^{*}$ Correspondence:

Jimena Leyria

jimenal.leyria@utoronto.ca

Specialty section:

This article was submitted to Invertebrate Physiology,

a section of the journal

Frontiers in Physiology

Received: 30 October 2020

Accepted: 18 January 2021

Published: 10 February 2021

Citation:

Leyria J, El-Mawed H, Orchard I and Lange $A B$ (2021) Regulation of a

Trehalose-Specific Facilitated

Transporter (TRET) by Insulin

and Adipokinetic Hormone

in Rhodnius prolixus, a Vector

of Chagas Disease.

Front. Physiol. 12:624165.

doi: 10.3389/fphys.2021.624165

\section{Regulation of a Trehalose-Specific Facilitated Transporter (TRET) by Insulin and Adipokinetic Hormone in Rhodnius prolixus, a Vector of Chagas Disease}

\author{
Jimena Leyria*, Hanine El-Mawed, lan Orchard and Angela B. Lange \\ Department of Biology, University of Toronto Mississauga, Mississauga, ON, Canada
}

Using the blood-sucking kissing bug, Rhodnius prolixus as an experimental model, we have studied the involvement of insulin-like peptides (ILPS) and adipokinetic hormone $(\mathrm{AKH})$ signaling in carbohydrate metabolism, focusing on the regulation of the trehalosespecific facilitated transporter (Rhopr-TRET), particularly in the ovaries. We find that trehalose stores in ovaries increase after feeding, synchronously with the beginning of vitellogenesis, but that the transcript expression of enzymes involved in trehalose synthesis show no changes between unfed and blood-fed animals. However, an eightfold increase in Rhopr-TRET transcript expression is observed in the ovaries post-blood meal. In vivo and ex vivo assays using exogenous insulins and Rhopr$A K H$, reveal that Rhopr-TRET is up-regulated in ovaries by both peptide families. In accordance with these results, when ILP and AKH signaling cascades are impaired using RNA interference, Rhopr-TRET transcript is down-regulated. In addition, trehalose injection induces an up-regulation of Rhopr-TRET transcript expression and suggests an activation of insulin signaling. Overall, the results support the hypothesis of a direct trehalose uptake by ovaries from the hemolymph through Rhopr-TRET, regulated by ILP and/or AKH. We also show that Rhopr-TRET may work cooperatively with AKH signaling to support the release of trehalose from the ovaries into the hemolymph during the unfed (starved) condition. In conclusion, the results indicate that in females of $R$. prolixus, trehalose metabolism and its hormonal regulation by ILP and AKH play critical roles in adapting to different nutritional conditions and physiological states.

Keywords: reproduction, starvation, triatominae, carbohydrate metabolism, hormonal regulation

\section{INTRODUCTION}

Carbohydrates represent one of the main energy reserves in animal cells, which is why they participate as essential factors in various biological processes of all multicellular organisms (Brosnan, 1999). In insects, glucose is stored in its polymeric form, glycogen, which is synthesized mainly from dietary carbohydrates or amino acids by the fat body, a multifunctional organ analogous to vertebrate adipose tissue and liver (Arrese and Soulages, 2010). Glycogen can be 
readily degraded on demand to be used as a glycolytic fuel, or mobilized from the fat body as trehalose for uptake by other tissues. Trehalose is a non-reducing disaccharide that consists of two glucose molecules and is non-toxic at the high levels that are found in insect hemolymph (Elbein et al., 2003). Trehalose biosynthesis depends mainly on two enzymatic reactions involving trehalose-6-phosphate synthase (TPS) and trehalose-6-phosphate phosphatase (Thompson, 2003). However, to be used in cell metabolism, trehalose must be converted into glucose by enzymes called trehalases (Shukla et al., 2015). A trehalose-specific facilitated transporter (TRET) leads to both the transfer of newly synthesized trehalose from the fat body into the circulating hemolymph and its uptake by other tissues, working in a reversible bidirectional way depending on the concentration gradient of trehalose and physiological needs (Thompson, 2003).

In insects, carbohydrate homeostasis is under strict hormonal regulation (Toprak, 2020) led mainly by insulinlike peptides (ILPs) and/or insulin-like growth factors (IGFs), and adipokinetic hormones (AKHs). The release and circulating levels of these peptide hormones are mainly regulated by feeding and starvation. In this context, the elevated circulating carbohydrate level after feeding is reduced by take-up into the fat body or ovaries through the actions of ILPs or IGFs to regulate anabolic processes, e.g., glycogen synthesis; whereas during starvation, defined as a prolonged unfed condition, catabolic processes are enhanced, e.g., glycogen breakdown and gluconeogenesis led mainly through $\mathrm{AKH}$ signaling (Arrese and Soulages, 2010; Badisco et al., 2013; Toprak, 2020).

Blood-sucking insects usually need to process a large amount of blood in a short time to provide energy to support the optimal progress of physiological events related to growth, development, and reproduction. They also often have to overcome long periods of starvation between successful gorging (Millen and Beckel, 1970; Foster, 1995). Insect reproduction is a process that places high demand on female metabolism for the provision of nutrients and other components for egg formation (Roy et al., 2018). Reproduction is therefore intimately associated with nutrition and metabolic state. While juvenile hormones (JHs) and ecdysteroids are the key players involved in the hormonal regulation of vitellogenesis to promote egg growth, insulin and $\mathrm{AKH}$ signaling have a critical role in controlling nutrient signaling and generating the energy necessary for those events to occur successfully (Roy et al., 2018; Lenaerts et al., 2019).

Triatomines (Hemiptera: Reduviidae) are a subfamily of blood-sucking insects with relevance in public health since they are vectors of Trypanosoma cruzi, the causal agent of Chagas disease. This illness is endemic in Latin American countries, but with globalization, this disease has spread throughout the world, and according to the World Health Organization, affects 6-7 million people worldwide (World Health Organization [WHO], 2020). Furthermore, due to climatic suitability, areas throughout the world have been identified as being at risk of becoming suitable habitats for the development of triatomines, thereby expanding endemic areas (Eberhard et al., 2020). In this context, research focusing on the physiology of triatomines can provide novel tools with potential for use in vector control strategies. Rhodnius prolixus, one of the most important vectors of Chagas disease, has been a classical model in insect physiology since the origins of the pioneering work of Wigglesworth (1936). R. prolixus blood-gorge only once in each instar and may remain for long periods of time in an unfed (starved) condition depending on availability of a blooddonating host.

In $R$. prolixus, AKH-signaling plays a critical role in modulating lipid levels (Marco et al., 2013; Patel et al., 2014; Zandawala et al., 2015; Alves-Bezerra et al., 2016), and the ILP/IGF cascade participates in lipid metabolism, carbohydrate mobilization and reproductive performance (Defferrari et al., 2016b, 2018; Leyria et al., 2020a). The presence of the AKH receptor $(\boldsymbol{A K H R})$ transcript and ILP/IGF signaling activation in ovaries of $\boldsymbol{R}$. prolixus has been reported (Zandawala et al., 2015; Alves-Bezerra et al., 2016; Leyria et al., 2020a), suggesting that both peptide hormones may also have a role in controlling aspects of reproductive physiology in ovaries. In addition, a transcriptome analysis of ovaries from fed females of $\boldsymbol{R}$. prolixus suggests that the trehalose which is taken up by that tissue comes from extra-ovarian sources (Leyria et al., 2020b). Although in the ovaries of $\boldsymbol{R}$. prolixus females, the percentage of carbohydrates stored during vitellogenesis is lower (around 6-10\%) compared to those found for other nutrients, such as lipids and proteins (Santos et al., 2008, 2012; Leyria et al., 2020b), the use of glycogen has been related to successful embryonic development, revealing the importance of carbohydrate accumulation by oocytes. Here, we present results supporting the hypothesis that a direct trehalose uptake through the $R$. prolixus TRET (Rhopr-TRET), regulated by insulin and/or AKH-signaling, is important for the storage of carbohydrates by the ovaries. Also, we demonstrate that Rhopr-TRET could work cooperatively with the $\boldsymbol{R}$. prolixus AKH (Rhopr-AKH) signaling pathway to promote the release of trehalose from the fat body and ovaries to the hemolymph during starvation. Overall, these results show that in $\boldsymbol{R}$. prolixus, carbohydrate metabolism and its hormonal regulation may play critical roles in adapting to different physiological conditions, including reproduction and starvation.

\section{MATERIALS AND METHODS}

\section{Insects}

Experiments were carried out with adults of $R$. prolixus taken from an established colony at the University of Toronto Mississauga. Insects were reared in incubators at $25^{\circ} \mathrm{C}$ under high humidity ( $\sim 50 \%)$. Standardized conditions of insect rearing were previously described (Leyria et al., 2020a). It is important to note that under our standardized conditions, female insects can only lay eggs after a blood meal (Leyria et al., 2020a,b). Thus, some experiments required females to take a blood meal in order to promote egg growth. For these experiments, females at 10 days post-ecdysis were fed through an artificial feeding membrane on defibrinated rabbit blood (Cedarlane Laboratories Inc., Burlington, ON, Canada). All insects used in this work have 
a similar feeding and body weight history. Tissues or hemolymph were sampled from adult females on representative days of the unfed and fed conditions, specified below for each experiment.

\section{Trehalose Quantification}

Adult female insects were dissected 10, 20, and 30 days postecdysis (representing the unfed condition, i.e., unfed as adults). One group of adult insects were fed and the tissues collected at $1,2,3,4$, and 5 days post-blood meal (representing the fed condition). Ovaries and ventral and dorsal fat bodies were dissected under cold $R$. prolixus saline $(150 \mathrm{mM} \mathrm{NaCl}, 8.6 \mathrm{mM}$ $\mathrm{KCl}, 2.0 \mathrm{mM} \mathrm{CaCl}_{2}, 8.5 \mathrm{mM} \mathrm{MgCl}_{2}, 4.0 \mathrm{mM} \mathrm{NaHCO}, 5.0 \mathrm{mM}$ HEPES, $\mathrm{pH} 7.0$ ) and then homogenized in $1.5 \mathrm{ml}$ microtubes containing $200 \mu \mathrm{l}$ of cold phosphate-buffered saline $(20 \mathrm{mM}$ $\mathrm{Na}_{2} \mathrm{HPO}_{4} / \mathrm{KH}_{2} \mathrm{PO}_{4}, 150 \mathrm{mM} \mathrm{NaCl}, \mathrm{pH}$ 6). The homogenates were centrifuged at $2,500 \times g$ for $5 \mathrm{~min}$ at $4^{\circ} \mathrm{C}$ and pellets containing tissue debris were discarded. The resulting material was re-centrifuged at $12,000 \times g$ for $10 \mathrm{~min}$ at $4^{\circ} \mathrm{C}$ and the supernatants collected for trehalose quantification. After immobilizing the insects on a slide using masking tape, $10 \mu \mathrm{L}$ of hemolymph was collected using a Hamilton syringe (Hamilton Company, Reno, NV, United States) from cut ends of the legs while pressing gently on the abdomen. The hemolymph was placed in ice-cold microtubes and then diluted in cold anticoagulant solution ( $10 \mathrm{mM} \mathrm{Na}{ }_{2}$ EDTA, $100 \mathrm{mM}$ glucose, $62 \mathrm{mM} \mathrm{NaCl}, 30 \mathrm{mM}$ sodium citrate, $26 \mathrm{mM}$ citric acid, $\mathrm{pH}$ 4.6) at a ratio of 1:5 (anticoagulant: hemolymph) (de Azambuja et al., 1991). Samples were then centrifuged at $10,000 \times g$ for $10 \mathrm{~min}$ at $4^{\circ} \mathrm{C}$ to remove hemocytes and the supernatants used for determination of trehalose concentration. Trehalose content in tissues and hemolymph was determined by the method of Parrou and François (1997) with slight modifications (Huang and Lee, 2011). In order to inactivate endogenous enzymes and to convert glucose into its reduced form, $50 \mu \mathrm{l}$ of tissue extract or $12.5 \mu \mathrm{l}$ of hemolymph (hemolymph + anticoagulant) were transferred to a new tube containing $50 \mu \mathrm{l}$ or $87.5 \mu \mathrm{l}$, respectively, of $0.25 \mathrm{M} \mathrm{Na}_{2} \mathrm{CO}_{3}$ buffer solution and incubated at $95^{\circ} \mathrm{C}$ for $10 \mathrm{~min}$. Once the tubes were cool, $80 \mu \mathrm{l}$ of $0.25 \mathrm{M}$ sodium acetate $(\mathrm{pH} 5.2)$ plus $20 \mu \mathrm{l}$ of $1 \mathrm{M}$ acetic acid were added, mixed and then centrifuged at $12,000 \times g$ for $10 \mathrm{~min}$ at room temperature. The supernatants obtained were used to measure trehalose levels. One-hundred $\mu \mathrm{l}$ of each supernatant was incubated overnight at $37^{\circ} \mathrm{C}$ with $1 \mu \mathrm{l}$ porcine kidney trehalase (Millipore-Sigma, Oakville, ON, Canada) to catalyze the conversion of trehalose into glucose, or with $R$. prolixus saline to determine the endogenous glucose content of the samples. Glucose contained in all the samples was determined using the glucose assay kit [Glucose (GO) Assay Kit, Millipore-Aldrich, Oakville, ON, Canada] according to the manufacturer's protocol $(30 \mu \mathrm{l}$ of sample was added to $100 \mu \mathrm{l}$ of glucose reagent solution). A standard curve using a $0-0.6 \mu \mathrm{g}$ range of glucose was run in parallel with the experimental samples. The samples were quantified at $540 \mathrm{~nm}$ using a plate reader spectrophotometer (Synergy HTX Multi-Mode Microplate Reader by Biotek, Winooski, VT, United States). The trehalose concentration was then corrected for the amount of glucose present in the samples (trehalose concentration $=$ (glucose measured in samples treated with trehalase - glucose measured in samples untreated)/2). The final trehalose concentration in each tissue or hemolymph sample was obtained by taking into consideration all dilutions used throughout the protocol. The results are shown as the mean \pm standard error of the mean $(\mathrm{SEM})(n=6$, where each $\mathrm{n}$ represents an individual tissue from 1 insect or a hemolymph pool from 2-3 insects).

\section{Identification and Cloning of cDNA Sequence Encoding the R. prolixus Trehalose-Specific Facilitated Transporter (Rhopr-TRET)}

By BLAST (Basic Local Alignment Search Tool) algorithm, the gene annotation from the RproC1.3 gene set (Mesquita et al., 2015) and using the trehalose transporter sequences from Nilaparvata lugens (XP_022183984.1) and Cimex lectularius (XP_024085685.1), we obtained the putative Rhopr-TRET. To successfully amplify and confirm the $5^{\prime}$ and $3^{\prime}$ regions of Rhopr-TRET, $5^{\prime}$ and $3^{\prime}$ rapid amplification of cDNA ends (RACE) assays were performed using the SMARTer RACE $5^{\prime} / 3^{\prime}$ Kit (Takara Bio USA, Mountain View, CA, United States). Briefly, fat bodies from fed females were dissected and placed in cold autoclaved phosphate buffered saline (PBS, $6.6 \mathrm{mM}$ $\mathrm{Na}_{2} \mathrm{HPO}_{4} / \mathrm{KH}_{2} \mathrm{PO}_{4}, 150 \mathrm{mM} \mathrm{NaCl}, \mathrm{pH}$ 7.4). Total RNA extraction was performed with Trizol reagent (Invitrogen by Thermo Fisher Scientific, MA, United States) according to the manufacturer's recommendations. The final concentration and A260/280 ratio of purified RNA was measured using a DS-11 + spectrophotometer (DeNovix Inc., Wilmington, DE, United States). All samples had a ratio between 1.9 and 2.0. RNA integrity, including potential degradation products and DNA contamination, was evaluated by electrophoresis in a $1 \%$ agarose gel (FroggaBio Inc., Concord, ON, Canada). RNA was considered intact when the $18 \mathrm{~S}$ rRNA band was observed. $5^{\prime}$ - and 3'-RACE-ready cDNA were synthesized using the SMARTScribe Reverse Transcriptase, provided in the kit. Three specific pairs of primers were used in order to ensure specificity of the amplified sequence. The manufacturer-supplied protocol was followed to perform RACE reactions. The final products of the RACE reactions were later subjected to $2 \%$ agarose gel electrophoresis and the single products obtained of the expected sizes were sent for Sanger sequencing by Macrogen USA (Macrogen, Brooklyn, NY, United States). The gene-specific primers utilized are shown in Supplementary Table 1. All reactions were performed using an s1000 thermal cycler (Bio-Rad Laboratories, Mississauga, ON, Canada).

\section{Sequence Analysis of Rhopr-TRET}

Following sequencing of Rhopr-TRET, the predicted structural and biochemical features of the transporter were analyzed using online tools. To predict the exon-intron boundaries within the sequence, nucleotide BLAST (BLASTn) was performed using the $R$. prolixus genome deposited in VectorBase. Exon-Intron 
Graphic Maker was the tool used to make the exon map of RhoprTRET with the ORF ${ }^{1}$. The deduced amino acid sequence and molecular mass prediction were assessed using tools available on ExPASy ${ }^{2}$ (SIB Bioinformatics Resource Portal) (Artimo et al., 2012). The transmembrane domains were predicted by TMHMM Server v.2.03 ${ }^{3}$ NetPhos 3.1 server was used to predict the potential intracellular phosphorylation sites ${ }^{4}$, NetNGlyc 1.0 server for predicting the potential N-linked glycosylation sites ${ }^{5}$, and GPSlipid for predicting lipid modifications ${ }^{6}$ (Blom et al., 1999; Sigrist et al., 2013; Xie et al., 2016). In order to provide insights into Rhopr-TRET function, the identification of potential domains was analyzed by Pfam 33.1 server $^{7}$ (El-Gebali et al., 2019). In addition, using VectorBase and orthoMCL DB (Li et al., 2003), potential orthologs were identified. RNA-seq data, available from the National Center for Biotechnology Information (NCBI) database (accession numbers PRJNA624187 and PRJNA624904 BioProjects) (Leyria et al., 2020a,b), was used to analyze the transcript expression of putative orthologs in the fat body and ovaries of unfed and fed $R$. prolixus females. Maxima structural model was built based on crystallographic data from proteins with similar secondary structure arrangements, using Phyre2 server (Kelley et al., 2015). The structure was visualized and analyzed using EZmol software (Reynolds et al., 2018).

\section{Phylogenetic Analysis of Rhopr-TRET}

Alignment of Rhopr-TRET was performed with TRET sequences from several invertebrate species, a bacterium and various glucose transporters from mammals, using the MUSCLE alignment tool $^{8}$ and later imported into the BOXSHADE 3.21 server ${ }^{9}$. Thirty-six amino acid sequences (Supplementary File 1) were imported into MEGA X (Molecular Evolutionary Genetics Analysis) (Pennsylvania, United States) (Kumar et al., 2018). To determine the relationship between insect sequences, a phylogenetic tree was constructed using the maximum likelihood method [based on the Jones-Thornton-Taylor (JTT) matrixbased model] (Jones et al., 1992). Bootstrap analysis was carried out using 1,000 replicates to determine confidence statistics (Felsenstein, 1985). In addition, a phylogenetic analysis of Rhopr-TRET and putative orthologs was performed as described above.

\section{RNA Extraction and Reverse Transcription/Quantitative PCR (RT-qPCR)}

mRNA levels of TPS (VectorBase: RPRC003010), Rhopr-TRET and 2 trehalases, membrane-bound $(m$-Tre) and soluble $(s-$ Tre) enzymes (VectorBase: RPRC004614 and RPRC012647,

\footnotetext{
${ }^{1}$ http://wormweb.org/exonintron

${ }^{2}$ www.expasy.org

${ }^{3}$ http://www.cbs.dtu.dk/services/TMHMM/

${ }^{4}$ http://www.cbs.dtu.dk/services/NetPhos/

${ }^{5} \mathrm{http} / / /$ www.cbs.dtu.dk/services/NetNGlyc/

${ }^{6} \mathrm{http}: / /$ lipid.biocuckoo.org/

${ }^{7}$ http://pfam.xfam.org/

${ }^{8} \mathrm{https} / / /$ www.ebi.ac.uk/Tools/msa/muscle/

${ }^{9}$ https://embnet.vital-it.ch/software/BOX_form.html
}

respectively), were assessed in the fat body and ovaries of females throughout different time points of the unfed and fed states. In addition, the Rhopr-AKH precursor (VectorBase: RPRC000416) and its receptor, Rhopr-AKHR (GenBank: KF534791), were analyzed throughout different time points of the unfed condition in fat body, ovaries and central nervous system [CNS, composed of the brain, the suboesophageal ganglion, the prothoracic ganglion and the mesothoracic ganglionic mass, and also including the corpora cardiaca/corpora allata complex (CC/CA)]. The spatial distribution of the Rhopr-TRET transcript in different tissues (CNS, ovaries, oviducts, fat body, foregut, anterior midgut, posterior midgut, salivary glands, accessory reproductive glands, hemocytes, hindgut, Malpighian tubules and dorsal vessel) was evaluated during the unfed condition (baseline stage). Tissue dissection was performed as previously reported (Leyria et al., 2020a,b). Briefly, the tissues were dissected from females during different time points of unfed and fed states, in cold autoclaved PBS. For hemocyte collection, hemolymph samples were obtained as stated in Section "Trehalose Quantification" and then the hemocytes were pelleted by centrifugation at $10,000 \times g$ for $10 \mathrm{~min}$ at $4^{\circ} \mathrm{C}$. Total RNA extractions were performed with Trizol reagent, as described above. cDNA synthesis was performed from $1 \mu \mathrm{g}$ of total RNA by reverse transcription reaction using random primers and $50 \mathrm{U}$ of MultiScribe MuLV reverse transcriptase (High Capacity cDNA Reverse Transcription Kit, Applied-Biosystems, by Fisher Scientific, ON, Canada). The conditions of the thermal cycler were: $10 \mathrm{~min}$ at $25^{\circ} \mathrm{C}, 120 \mathrm{~min}$ at $37^{\circ} \mathrm{C}$, and $5 \mathrm{~min}$ at $85^{\circ} \mathrm{C}$. qPCR assays were performed using an advanced master mix with super green low rox reagent (Wisent Bioproducts Inc., QC, Canada), according to the manufacturer's recommendations, using 4 pmol of sense and antisense primers in a final volume of $10 \mu \mathrm{l}$. The qPCR temperature-cycling profile was: initial denaturation $3 \mathrm{~min}$ at $95^{\circ} \mathrm{C}$, followed by 39 cycles of $30 \mathrm{~s}$ at $94^{\circ} \mathrm{C}, 30 \mathrm{~s}$ at $58-$ $60^{\circ} \mathrm{C}$ (depending on the pair of primers used), and $1 \mathrm{~min}$ at $72^{\circ} \mathrm{C}$, followed by a final extension at $72^{\circ} \mathrm{C}$ for $10 \mathrm{~min}$. Each reaction contained 3 technical replicates as well as a no template control and a no reverse transcriptase control. The reactions were carried out using a CFX384 Touch Real-Time PCR Detection System (Bio-Rad Laboratories Ltd., ON, Canada). Quantitative validation was analyzed by the $2^{-\Delta \Delta} \mathrm{Ct}$ method (Livak and Schmittgen, 2011). 18S ribosomal RNA subunit and $\beta$-actin were used as reference genes (Majerowicz et al., 2011; Leyria et al., 2020a) and the transcript expressions were normalized via geometric mean. Primers (by Sigma-Aldrich, ON, Canada) are listed in Supplementary Table 1. Primers were designed at the exon-exon junction (i.e., over two different exons) in order to ensure that the primers only amplify transcripts. For each pair of primers, the efficiency ranged from 88 to $116 \%$, with linear correlation coefficients $\left(r^{2}\right)$ ranging from 0.8 to 1 , and the dissociation curves always showed a single peak, indicating that a single cDNA product was amplified and excluding the possibility or tendency of the primers to form dimers. Specific target amplification was confirmed by automated DNA sequencing, as described above. The results are shown as the mean \pm SEM ( $n=3-4$, where each $n$ represents a pool of tissues from 3 insects). 


\section{Ex vivo and in vivo Insulin and $\mathrm{AKH}$ Signaling Stimulation}

For ex vivo assays, ovaries of unfed females at 10 days post-ecdysis were incubated with human insulin (MilliporeSigma, Oakville, ON, Canada), porcine insulin (Millipore-Sigma, Oakville, ON, Canada), $R$. prolixus ILP1 (Rhopr-ILP1, chemically synthesized by DGpeptidesCo., Ltd., Zhejiang, China) or with of the mammalian insulin receptor-specific activator bpV (phen) (Millipore-Sigma, Milwaukee, WI, United States). Insulin stock solutions were solubilized in $\mathrm{pH} 3$ with $\mathrm{HCl}$ and $\mathrm{bpV}$ (phen) in dimethyl sulfoxide (DMSO), according to the manufacturer protocol. To make working solutions, all the drugs were diluted in $R$. prolixus saline. The incubations were performed for $3 \mathrm{~h}$ or $12 \mathrm{~h}$, as indicated, in $200 \mathrm{ml}$ of Grace's Insect Medium (Millipore-Sigma, Oakville, ON, Canada), at $30^{\circ} \mathrm{C}$ in the dark, as previously described (Cruz et al., 2006). The final concentration of human insulin, porcine insulin and Rhopr-ILP1 in the incubation medium was $1 \mathrm{mM}$, and for bpV(phen) was $0.1 \mathrm{mM}$. The stimulatory effects of mammalian insulins and bpV (phen) on the insulin receptor pathway in $R$. prolixus were previously reported (Defferrari et al., 2018; Leyria et al., 2020a). In parallel, ovaries of unfed females at 10 days post-ecdysis were incubated under the same condition with $0.1 \mu \mathrm{M} R$. prolixus AKH (RhoprAKH, pQLTFSTDWamide, chemically synthesized by Genscript Laboratories, Piscataway, NJ, United States). Stock solutions of Rhopr-AKH were dissolved in 50\% 1-Methyl-2-Pyrrolidinone (Millipore-Sigma, Oakville, ON, Canada) and then stored at $-20^{\circ} \mathrm{C}$ (Zandawala et al., 2015; Hamoudi et al., 2016). RhoprAKH was diluted with $R$. prolixus saline prior to performing the experiments. The solvent in the working dilution represents only $0.001 \%$ of the final volume; previously we demonstrated that this concentration does not interfere with experiments (Zandawala et al., 2015). After incubation, all tissues were immediately placed in Trizol and processed for RNA extraction and subsequent RTqPCR to assess Rhopr-TRET and TPS mRNA levels. For both ex vivo assays, the results are shown as the mean $\pm \operatorname{SEM}(n=3-4$, where each $n$ represents a pool of tissues from 2 insects).

For in vivo assays, unfed insects (10 days post-ecdysis) were injected into the hemocoel with $5 \mu \mathrm{l}$ of $0.1 \mu \mathrm{g} / \mu \mathrm{l}$ porcine insulin (Defferrari et al., 2018; Leyria et al., 2020a) or $0.01 \mu \mathrm{g} / \mu \mathrm{l}$ RhoprAKH (Zandawala et al., 2015). As control, a group of insects was injected with $5 \mu \mathrm{l}$ of $R$. prolixus saline. Ovaries were removed under autoclaved PBS at $3 \mathrm{~h}$ post-injection and processed for RNA extraction and subsequent RT-qPCR to assess Rhopr-TRET and TPS mRNA levels. We used females at 10 days post-ecdysis for in vivo assays because (a) we previously demonstrated that unfed females of $R$. prolixus are in a sensitized state to respond to an increase of ILP levels by rapidly activating ILP signaling (Leyria et al., 2020a), and (b) food deprivation is the main stimulus for the release of $\mathrm{AKH}$, which works as a metabolic stimulator leading to both carbohydrate and lipid mobilization (Bednáøová et al., 2013; Alves-Bezerra et al., 2016) and, thus the tissues should be also sensitized to respond rapidly to $\mathrm{AKH}$ stimulation. For both in vivo assays, the results are shown as the mean $\pm \operatorname{SEM}(n=3-4$, where each $n$ represents a pool of tissues from 2 insects).

\section{Double-Stranded RNA (dsRNA) Design and Synthesis}

In order to downregulate insulin and AKH signaling, the specific receptors involved in the activation of their respective cascades were interfered with using dsRNA. Gene specific primers were combined with the T7 RNA polymerase promoter sequence (Supplementary Table 1). Briefly, the following temperaturecycling profile was used for all PCRs: initial denaturation at $94^{\circ} \mathrm{C}$ for $3 \mathrm{~min}$, followed by 30 cycles of $94^{\circ} \mathrm{C}$ for $30 \mathrm{~s}, 58^{\circ} \mathrm{C}$ for $30 \mathrm{~s}$ and $72^{\circ} \mathrm{C}$ for $90 \mathrm{~s}$, and a final extension at $72^{\circ} \mathrm{C}$ for $10 \mathrm{~min}$. The final PCR product was purified and used as template to synthesize dsRNA with T7 Ribomax Express RNAi System (Promega, Madison, WI, United States), according to the manufacturer's recommendations. As control, a dsRNA molecule based on the Ampicillin Resistance Gene (dsARG) from the pGEM-T Easy Vector system (Promega, Madison, WI, United States) was used throughout the study (Leyria et al., 2020a). The dsRNAs obtained were subjected to $2 \%$ agarose gel electrophoresis. A single product of the expected size was obtained for each dsRNA, which was sequenced, as described above, to confirm specificity. The sequences obtained were subjected to BLAST analysis in order to search for possible targets on VectorBase, where the $R$. prolixus genome is deposited. Alignments with the bestmatching sequences are shown and scored in Supplementary Figure 1. Only 1 high confidence hit, i.e., genomic regions identified with high similarity with our sequence, was obtained. In addition, the specificity of dsRhopr-IR1 and dsRhopr-AKHR to downregulate Rhopr-IR1 (Vectorbase: RPRC006251) and Rhopr-AKHR (GenBank: KF534791), respectively, was previously demonstrated (Zandawala et al., 2015; Defferrari et al., 2018).

\section{Knockdown of Transcript Expression Using Double Stranded RNA}

To evaluate the potential regulation of insulin signaling on Rhopr-TRET expression in ovaries, we used insects from a fed state, a condition where we previously demonstrated that the insulin cascade is activated (Leyria et al., 2020a). In brief, females at 10 days post-ecdysis were injected into the hemocoel with $5 \mu \mathrm{L}$ of $2 \mu \mathrm{g} / \mu \mathrm{L}$ of dsRhopr-IR or dsARG and $3 \mathrm{~h}$ later the animals were given a blood meal. Insects were dissected 3 days post-injection and the transcriptional expression to Rhopr-IR and Rhopr-TRET were measured in the tissues by RT-qPCR, as described above. To evaluate the role of $\mathrm{AKH}$ signaling on Rhopr-TRET transcriptional expression, we used insects from an unfed state, a condition where AKH signaling should be increased. In brief, females 10 days post-ecdysis were injected into the hemocoel with $5 \mu \mathrm{L}$ of $2 \mu \mathrm{g} / \mu \mathrm{L}$ of dsRhopr-AKHR or dsARG. Insects were dissected 1 or 2 days post-injection and the transcriptional expression of Rhopr-AKHR and RhoprTRET were measured in tissues by RT-qPCR, as described above. Groups of dsRhopr-IR1, dsRhopr-AKHR and dsARG treated insects (unfed females) were separated and injected (1 day after dsRNA treatment) into the hemocoel with $5 \mu \mathrm{l}$ of $0.1 \mu \mathrm{g} / \mu \mathrm{l}$ porcine insulin, $0.01 \mu \mathrm{g} / \mu \mathrm{l}$ Rhopr-AKH or $R$. prolixus saline (control). Ovaries were removed under autoclaved PBS at $3 \mathrm{~h}$ after hormone injection and processed to RNA extraction and 
subsequent RT-qPCR to assess Rhopr-IR1, Rhopr-AKHR and Rhopr-TRET mRNA levels, as indicated. In all cases, the results are shown as the mean \pm SEM $(n=4-5$, where each $n$ represents an individual tissue from 1 insect). In addition, the percent survival of dsRhopr-AKHR injected insects during the unfed condition was analyzed $(n=15)$.

\section{In vivo Trehalose Injection}

In order to evaluate the effect of an increase in circulating trehalose on insulin and AKH signaling, unfed females (10 days post-ecdysis) were injected into the hemocoel with $5 \mu \mathrm{l}$ of $70 \mu \mathrm{g} / \mu \mathrm{l}$ trehalose solution (Millipore-Sigma, Oakville, ON, Canada) diluted in $R$. prolixus saline, or $5 \mu$ l of $R$. prolixus saline (control). Insects were dissected $3 \mathrm{~h}$ post-injection and the expression of Rhopr-TRET and of the peptides Rhopr-ILP1 (GenBank: KT896507.1), Rhopr-IGF (R. prolixus insulin growth factor, GenBank: KX185519.1) and Rhopr-AKH were measured in the ovaries, fat bodies or CNS, as indicated, by RT-qPCR. In addition, groups of dsRhopr-IR1 and dsARG treated insects were injected (1 day after dsRNA treatment) into the hemocoel with $5 \mu \mathrm{l}$ of $70 \mu \mathrm{g} / \mu \mathrm{l}$ trehalose solution or $5 \mu \mathrm{l}$ of $R$. prolixus saline (control). Fat bodies were removed under autoclaved PBS at $3 \mathrm{~h}$ post-injection and processed for RNA extraction and subsequent RT-qPCR to assess Rhopr-IR1 and Rhopr-TRET mRNA levels. In all cases, the results are shown as the mean \pm SEM $(n=4-5$, where each $\mathrm{n}$ represents an individual tissue from 1 insect).

\section{Statistical Analyses}

All graphs were created using GraphPad Prism 9 (GraphPad Software, San Diego, CA, United States ${ }^{10}$ ). Before performing statistical analysis, we evaluated the data for normality and homogeneity of variance using the Shapiro-Wilk test, which showed that no transformations were needed, all datasets passed

${ }^{10}$ www.graphpad.com normality and homoscedasticity tests. Multiple group analysis was conducted by one-way ANOVA and Tukey's test as post hoc test, and statistically significant difference between two groups were inferred using $T$-test. Differences in the survival curve were analyzed using the Log-Rank test. In all the cases, a $p$-value $<0.05$ was considered statistically significant.

\section{RESULTS}

\section{Trehalose Quantification in Fat Body, Ovaries, and Hemolymph in Response to a Blood Meal}

Trehalose content in key tissues involved in reproduction, namely the fat body (Figure 1A) and ovaries (Figure 1B), as well as circulating titers in the hemolymph (Figure 1C), was measured at representative days during the unfed and fed conditions. Under our experimental conditions, no trehalose is detected in the fat body or the ovaries at 10 and 20 days post-ecdysis; indeed, it remains undetectable in the ovaries until feeding. Following the blood meal, there is an overall increase in trehalose content in both tissues as well as in the hemolymph. While the trehalose content in the fat body and ovaries is highest at 5 days post-blood meal, the highest hemolymph content is seen on the fourth day and remains at this high level on the fifth day after feeding.

\section{Rhopr-TRET cDNA Sequence, Characterization, and Modeling}

Recently, by transcriptome analysis we showed that RhoprTRET is more than six and threefold up-regulated in ovaries and fat body of fed insects, respectively (Leyria et al., 2020b), supporting the hypothesis that a direct trehalose uptake from the hemolymph via Rhopr-TRET could be an important process

\section{Trehalose quantification}

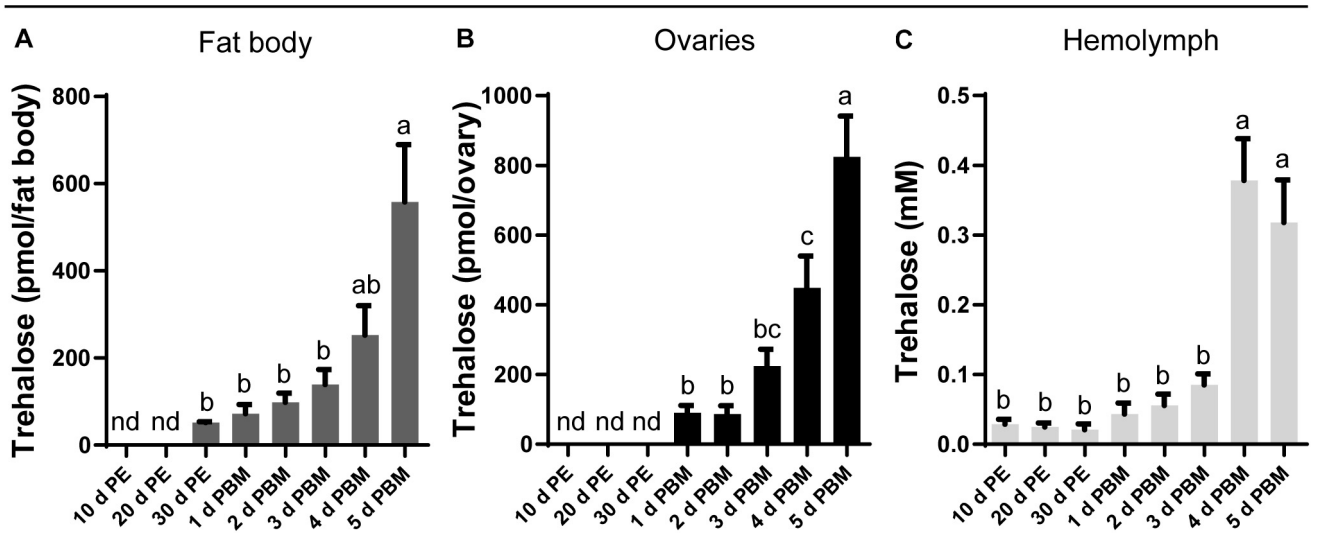

FIGURE 1 | Trehalose content in the fat body, ovary, and hemolymph of unfed and fed $R$. prolixus females. The trehalose content of fat body (A), ovaries (B), and hemolymph (C) was quantified in unfed adult females [10, 20, and 30 days post-ecdysis (PE)] and in fed adult females at 1, 2, 3, 4, and 5 days post blood meal (PBM). Trehalose content increases significantly in both tissues and hemolymph following a blood-meal. The results are shown as the mean \pm SEM $(n=6$, where each $n$ represents an individual tissue from 1 insect or a hemolymph pool from 2 to 3 insects). Statistically significant differences were determined by a one-way ANOVA and a Tukey's test as the post hoc test. Different letters indicate significant difference at $P<0.05$. nd, not detected. 
involved in the storage of carbohydrates in ovaries. The RhoprTRET candidate sequence was identified within the $R$. prolixus genome under the reference RPRC007957 and annotated as a partial sequence. We used this partial predicted sequence to design primers for RACE in order to confirm and obtain the complete sequence (GenBank: MW196439.1). Our results by RACE show an open reading frame (ORF) to Rhopr-TRET of $1,461 \mathrm{bp}$, which encodes a $51.58-\mathrm{kDa}$ protein of 477 amino acids (Supplementary Figure 2). The ORF spans 6 exons, separated by 5 introns (Figure $\mathbf{2 A}$ ). The domain prediction analysis revealed that Rhopr-TRET belongs to the sugar-transporter superfamily, including in its sequence the major facilitator superfamily domain, (MFS: CL0015) (Supplementary Figure 3). The RPRC007957 protein was previously annotated on the UniprotKB database (T1HV87_RHOPR) under the general classification of MFS. In addition, 12 sequences deposited on VectorBase were identified with the same conserved MFS domain, all of them belonging to the sugar transporter family (PF00083). Using BLASTp algorithm, 6 of them were classified as putative glucose transporters (RPRC002241, RPRC004616, RPRC010167, RPRC010168, RPRC011096, and RPRC014535), 1 was classified as a ribonuclease $\mathrm{P}$ protein subunit p25-like protein (RPRC011469), and 5 as putative trehalose transporters (RPRC002688, RPRC002833, RPRC005613, RPRC011385, and RPRC015169) (Supplementary File 2). Indeed, the phylogenetic tree groups Rhopr-TRET with the other putative trehalose transporters, suggesting the existence of a close relationship between them. In addition, all of the putative glucose transporters form a separate monophyletic group (Supplementary Figure 4). The alignment of the Rhopr-TRET amino acid sequence (via the online tool MUSCLE) with all of these sequences is shown in Supplementary File 3. RNA-seq data analysis shows that the putative trehalose transporters are, in general, more highly expressed in both tissues than are the glucose transporters, with Rhopr-TRET (RPRC007957) having the highest fold changes between fed and unfed insects (Supplementary File 2); and so subsequently this transcript was chosen to analyze in depth in this work. The putative transmembrane domains were predicted and suggest that Rhopr-TRET forms a 12-transmembrane structure, with a long loop connecting the transmembrane domain 6 (TM6) and TM7 on the cytosolic side of the membrane, and an intracellular $N$ - and $C$-terminus, which are typical characteristics for the MFS (Figure 2B). Furthermore, residues conserved across all MFS members, with functional significance, are observed and highlighted (Figure 2B) (Kanamori et al., 2010; Price et al., 2010): tryptophan (W) and proline (P) residues in TM10, which are involved in substrate selectivity and conformational flexibility, respectively; QLS motif in TM7, involved in transport activity; glycine $(G)$ residues conserved in the TM 1, 4, 5, 7,8 , and 10 , critical to stabilize the structure; GRR/K motif in the second loop, a position characteristic of members of MFS, and a tryptophan residue in TM11, essential for transport activity (Kanamori et al., 2010), among others. By homology modeling, we can correctly predict the target structure of RhoprTRET (Figure 2C). In general, we note that polar residues form a central aqueous channel through which trehalose could be translocated through the membrane, and the hydrophobic residues would be those that participate in the anchoring of the transporter to the membrane since they are observed in a greater density on the side facing the membrane, outside of the channel (Figure 2C). Also, we observe post-translational modifications to Rhopr-TRET which would ensure complete functionality (Figure 2D): $N$-glycosylation is predicted to occur at Asn135, and Asn265 and Asp317, phosphorylation sites are predicted on $14 \mathrm{Thr}$ residues, $6 \mathrm{Tyr}$ residues, and $28 \mathrm{Ser}$ residues, and palmitoylation predicted to occur on Cys149. Interestingly, we were unable to find the typical motif of most MFS sequences, an $N$-glycosylation site in the first extracellular loop (between TM1 and TM2).

As part of the characterization of Rhopr-TRET, we evaluated the spatial transcript expression of this transporter using various tissues from unfed adult females (baseline condition) using RT-qPCR. In accordance with the premise that the fat body is the main tissue involved in nutrient and energy metabolism, the highest expression of the Rhopr-TRET transcript is observed in this tissue (Figure 2E). The Rhopr-TRET amino acid sequence was aligned via the online tool MUSCLE with a number of sequenced orthologs from other species, including those for glucose transporters of mammals and a TRET sequence from a bacterium (Supplementary File 1). The results highlight the high degree of conservation in TRET sequences from insects. Indeed, the phylogenetic tree groups Rhopr-TRET with other hemipterans (Supplementary Figure 3), suggesting the existence of a close relationship to both the bed bug ( $C$. lectularius) and the brown marmorated stink bug (Halyomorpha halys). In addition, all the glucose transporters form a separate monophyletic group and the TRET from Escherichia coli (WP_077788896.1) forms the out group.

\section{Transcript Expression in Response to a Blood Meal}

In order to obtain a general view on the dynamics of trehalose metabolism, it is essential to evaluate the expression of transcripts involved in the control of trehalose homeostasis. We examined the profiles of transcript expression of TPS, $m$-Tre and $s$-Tre as well as of Rhopr-TRET in the fat body and ovaries. Except for TPS, the transcript expression pattern in both tissues is quite similar throughout the different time points of the unfed and fed condition. In the fat body, TPS transcript levels are lower during the unfed condition but rise significantly post-blood meal when vitellogenesis begins, with maximum seen 1 day after feeding (Figure 3A). RhoprTRET transcript levels are also lower in the unfed condition but increase significantly $(\sim 10$-fold $)$ by day 1 after blood feeding and remain high over the 5 days analyzed (Figure 3B). $m$-Tre transcript expression has a non-specific pattern of expression with regard to blood feeding, with fluctuating levels throughout all the time points (Figure 3C). However, s-Tre transcript expression is higher at day 20 and 30 of the unfed condition and remains low after the blood meal but with a slight non-statistical increase as the days post-feeding advance (Figure 3D). 
A

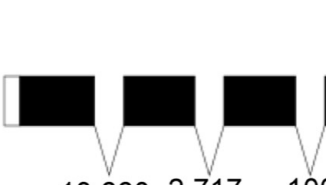

$13,660 \quad 2,717 \quad 100$

The exon map of Rhopr-TRET

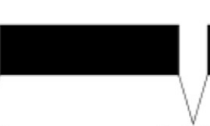

1,064

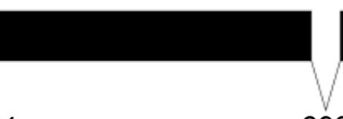

693

Putative transmembrane domains

B

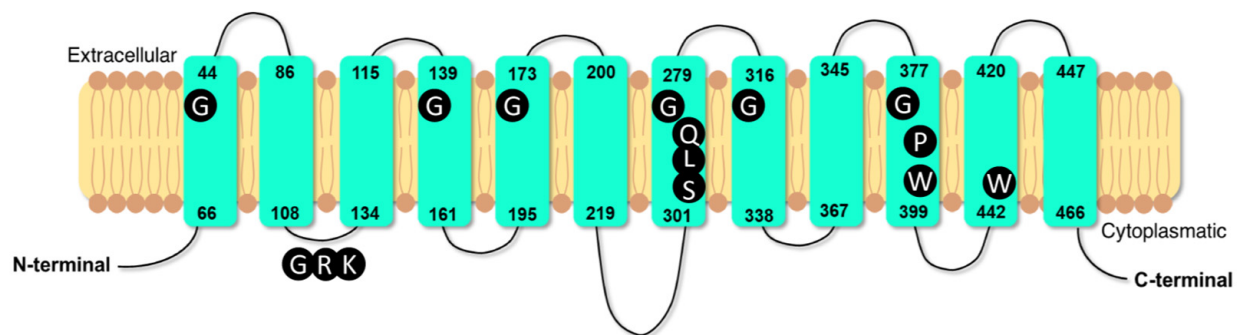

C

Rhopr-TRET protein model

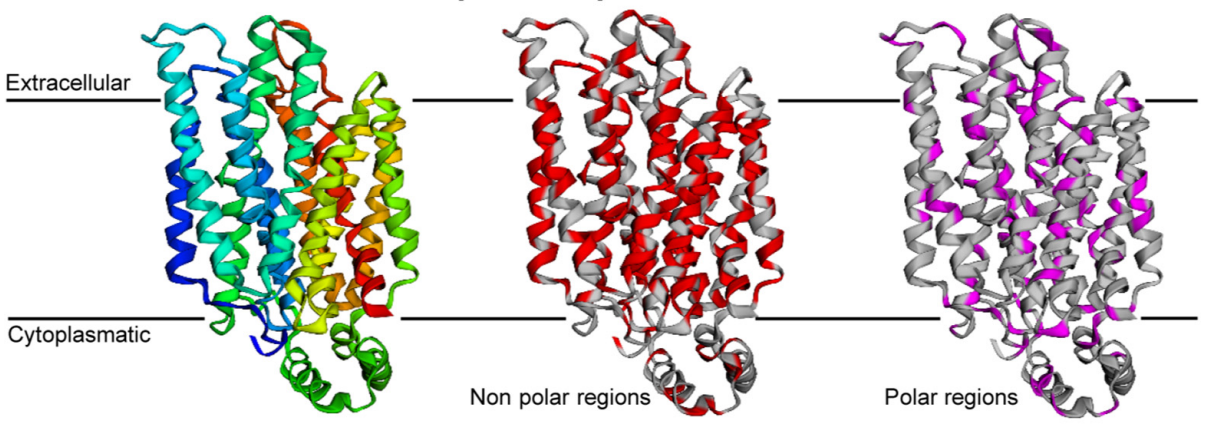

D Putative post-translational modifications

\begin{tabular}{c} 
Prediction/position \\
S-Palmitoylation sitel \\
149 \\
N-linked glycosylation sites/ \\
$135,265,317$ \\
$2,20,23,35,49,60,65,70,76,86,90,95$, \\
\hline Y-phosphorylation sites/ \\
$40,63,366,377,388,435$ \\
\hline S-phosphorylation sites/ \\
$126,137,144,154,237,253,258,267$, \\
$268,312,319,370,415,418,458,473$ \\
\hline T-phosphorylation sites $/$ \\
$109,115,119,172,178,221,259,303$, \\
$313,363,375,428,469,470$
\end{tabular}

E Tissue specific expression of Rhopr-TRET

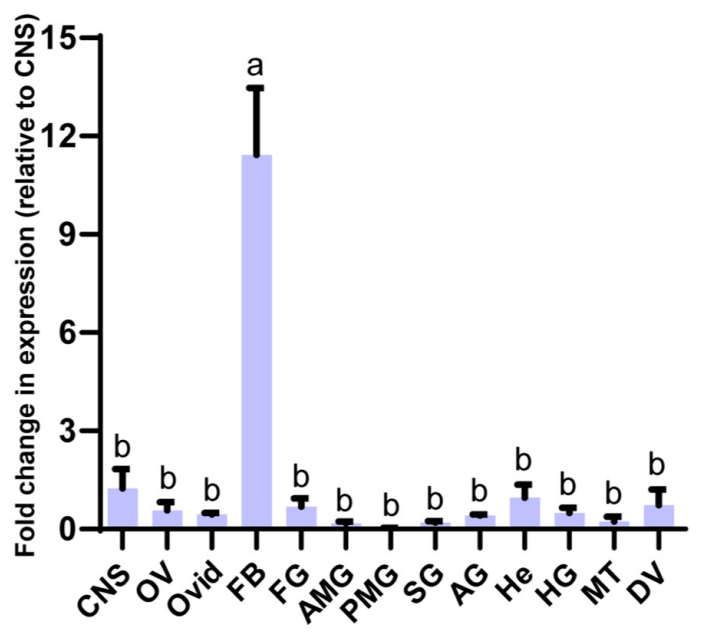

FIGURE 2 | Rhopr-TRET characterization and modeling. (A) Exon map of Rhopr-TRET of the open reading frame (ORF) and exons/introns. The ORF of Rhopr-TRET is denoted by the solid black box spanning the exons. The length of each box is representative of the nucleotide number (bar = $100 \mathrm{bp}$ ) and the numbers below represent the intron length as base pairs (bp). (B) Schematic diagram of Rhopr-TRET. The 12 transmembrane domains within the cell membrane and the position of the amino acids indicating the start and end of each transmembrane regions are shown. Conserved motifs among Rhopr-TRET sequences are highlighted in dark in their typical positions. (C) Molecular model of Rhopr-TRET. Cartoon representation of Rhopr-TRET illustrating the folds of the transporter; the putative non-polar and polar regions are also displayed. (D) Putative post-translational modifications, including N-glycosylation, phosphorylation and palmitoylation sites. (E) Distribution of Rhopr-TRET transcript in unfed adult female R. prolixus; central nervous system (CNS), ovaries (OV), oviducts (Ovid), fat body (FB), foregut (FG), anterior midgut (AMG), posterior midgut (PMG), salivary glands (SG), accessory glands (AG) (spermatheca and cement gland), hemocytes (He), hindgut (HG), Malpighian tubules (MT) and dorsal vessel (DV). The expression of transcripts in each tissue was quantified by RT-qPCR. The $y$-axis represents the fold change in expression relative to CNS (value $\sim 1)$. The results are shown as the mean \pm SEM $(n=3-4$, where each $n$ represents a pool of tissues from 3 insects). Statistically significant differences were determined by a one-way ANOVA and a Tukey's test as the post hoc test. Different letters indicate significant difference at $P<0.05$. 


\section{Temporal expression in fat body}

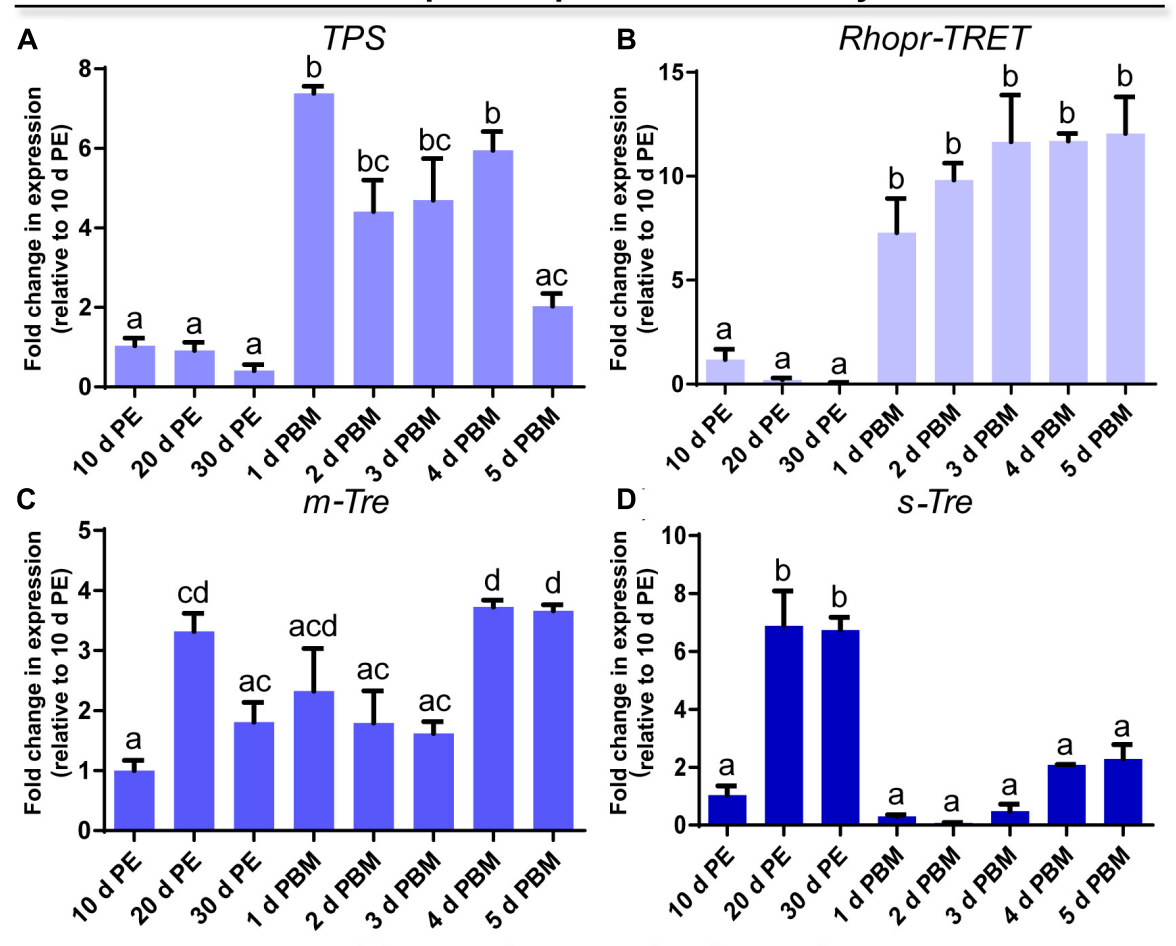

Temporal expression in ovaries

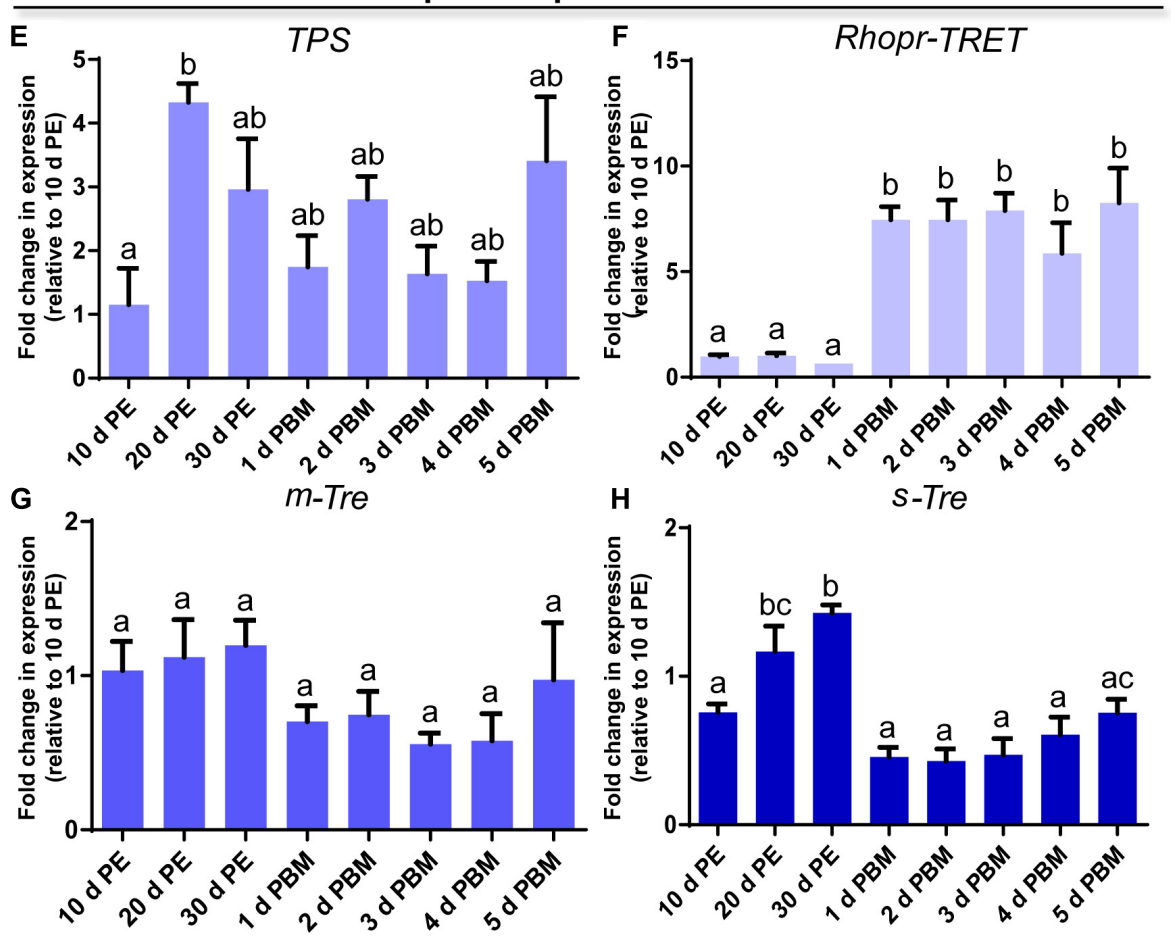

FIGURE 3 | Temporal expression of trehalose synthase (TPS), Rhopr-TRET, membrane-bound trehalase ( $m$-Tre), and soluble trehalase (s-Tre) in the fat body and ovaries of $R$. prolixus under different nutritional conditions. Transcript expression of TPS, Rhopr-TRET, $m$-Tre, and s-Tre was quantified by RT-qPCR in the fat body (A-D, respectively) and ovaries (E-H, respectively) of adult females at varying days post-ecdysis (d PE), representing unfed conditions, and various days post-blood meal (d PBM), representing the fed condition. Results were analyzed by the $\Delta \Delta C$ t method. The $y$-axis represents the fold change in expression relative to 10 days $\mathrm{PE}$ (value $\sim 1)$. The results are shown as the mean $\pm \operatorname{SEM}(n=3-4$, where each $n$ represents a pool of tissues from 3 insects). Statistically significant differences were determined by a one-way ANOVA and a Tukey's test as the post hoc test. Different letters indicate significant difference at $P<0.05$. 
In the ovaries, the transcript for TPS after a blood meal shows levels similar to those during the unfed condition, i.e., 10, 20, and 30 days post-ecdysis (Figure 3E). However, a significant upregulation of Rhopr-TRET transcript expression occurs after a blood meal when vitellogenesis has begun (Figure 3F). As seen in the fat body, $m$-Tre transcript expression has a fluctuating pattern throughout all the days analyzed (Figure 3G) and s-Tre transcript expression is up-regulated in unfed females (Figure $\mathbf{3 H}$ ).

\section{The Effects of Exogenous Insulins and Rhopr-AKH on Rhopr-TRET Transcript Expression in Ovaries From Unfed \\ Females}

Since insulin and $\mathrm{AKH}$ families are known to regulate carbohydrate metabolism in insects, we performed ex vivo and in vivo experiments using exogenous hormones and unfed female adults, where Rhopr-TRET transcript expression in the ovaries is low. For the ex vivo experiments, ovaries were incubated with insulin from 3 different sources, human insulin (H_In), porcine insulin (P_In), and R. prolixus ILP1 (Rhopr-ILP1), and an insulin receptor kinase activator, bpV (phen). RT-qPCR reveals that after $3 \mathrm{~h}$ of incubation, only the treatment with bpV (phen) is able to induce a significant increase in Rhopr-TRET expression ( $p<0.05$ vs. saline, by one-way ANOVA, $n=3-4$ ) (Figure 4A). However, after $12 \mathrm{~h}$ of incubation, P_In and Rhopr-ILP1 also induce a statistical increase in Rhopr-TRET transcript expression ( $p<0.05$, by one-way ANOVA, $n=3-4$ ) (Figure 4B). Rhopr$\mathrm{AKH}$ is also able to promote an up-regulation of Rhopr-TRET transcript expression in ovaries with $3 \mathrm{~h}$ of incubation $(p=0.019$ vs. saline, by Student's $t$-test, $n=3-4$ ) (Figure 4D), suggesting that Rhopr-TRET transcript expression is regulated by hormones and that the ovaries of unfed females are more sensitized to respond rapidly to Rhopr-AKH than to insulins. In the same

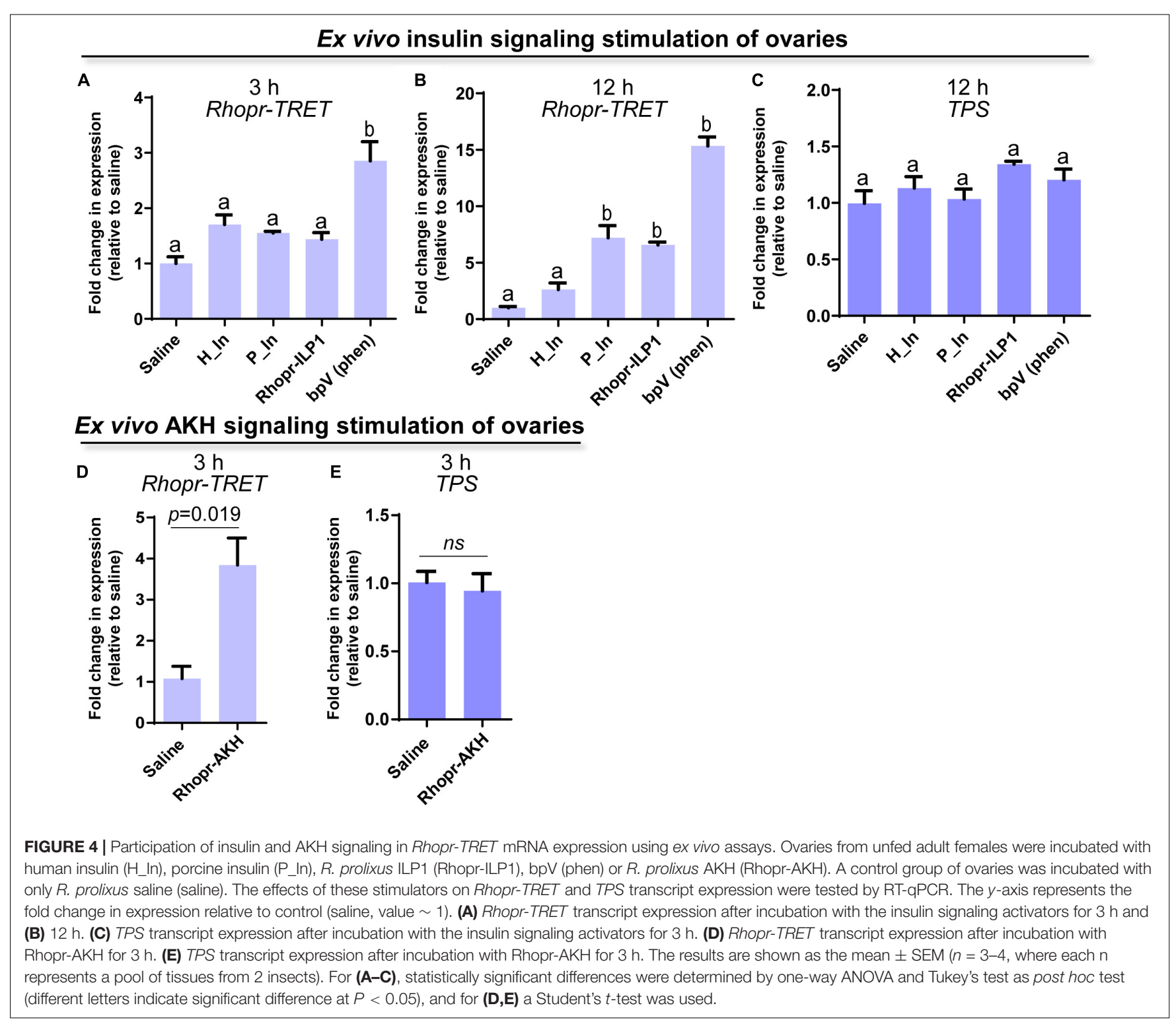


ovaries, TPS expression was measured in order to assess the specificity of the transcript stimulation. The results show that under these experimental conditions, the transcript levels of the enzyme involved in trehalose synthesis is not altered after incubation of the ovaries with insulins, bpV (phen) or RhoprAKH (Figures 4C,E, respectively). To better understand the role of Rhopr-AKH and insulin signaling on the expression of RhoprTRET in the ovaries, we upregulated AKH (using Rhopr-AKH) and insulin (using $\mathrm{P}_{\text {_In }}$ ) signaling in unfed females by injection of these hormones into females at 10 days post-ecdysis, when Rhopr-TRET expression in the ovaries is low (Figure 5A). At $3 \mathrm{~h}$ post-injection, both hormones significantly increased the expression of Rhopr-TRET mRNA in ovaries (Figures 5B,D). As with the ex vivo assays, neither Rhopr-AKH nor P_In injections altered TPS transcript expression in the ovaries at our experimental times (Figures 5C,E).

\section{Knockdown of Rhopr-IR1 and Rhopr-AKHR Transcripts and Effects on Rhopr-TRET mRNA Expression in Ovaries}

To further confirm the involvement of insulin and AKH signaling in regulating Rhopr-TRET transcript expression, we knocked down the receptors Rhopr-IR1 and Rhopr-AKHR, using RNAi in fed or unfed females (Figure 6A). The dsRhopr-IR1 treatment did not influence the survival of the insects over the time course of the experiments (data not shown). However, after 2 days post-treatment, the percent survival in dsRhopr-AKHR treated insects decreased by $80 \%$ with respect to controls (Figure 7D); as a consequence, the tissue collection was performed 1 or 2 days post-treatment. The live insects treated with dsRhoprAKHR showed behaviors, i.e., walking and movement of legs and proboscis when immobilized, comparable to controls at 2 days post-injection. In addition, to investigate the effects of dsRhoprIR1 knockdown on the amount of blood ingested, the insects were weighed before and after feeding and no significant difference between treated and control females was observed (data not shown). RT-qPCR reveals that transcript levels for Rhopr-IR1 and Rhopr-AKHR are reduced by 55 and $90 \%$, respectively, in ovaries, at 3 and 2 days, respectively, following RNAi treatment (Figures 6B,D). Knocking down either Rhopr-IR1 or RhoprAKHR transcripts reduced Rhopr-TRET transcript expression (Figures 6C,E). In addition, TPS transcript expression in the ovaries of these insects is not altered (data not shown). To confirm the potential regulation of Rhopr-TRET by hormones, we down-regulated Rhopr-IR1 and Rhopr-AKHR transcripts in unfed females and 1 day after dsRNA treatment, we injected porcine insulin (P_In) or Rhopr-AKH, as indicated (Figure 6F). Insects with down-regulated receptors (Figures 6G,I) are not able to increase Rhopr-TRET expression in ovaries after P_In (dsRhopr-IR1/P_In) or Rhopr-AKH (dsRhopr-AKHR/Rhopr$\mathrm{AKH})$ stimulation to the same levels as those of control insects [dsARG/P_In and dsARG/Rhopr-AKH (Figures 6H,J)]. Interestingly, dsRhopr-IR1 treated unfed insects (dsRhoprIR1/S), are not able to down-regulate Rhopr-TRET (Figure 6H) as occurs in dsRhopr-IR1 treated insects 3 days postfeeding (Figure 6C). This finding shows that the blood meal is an important signal to control Rhopr-TRET mRNA levels via insulin signaling.

\section{The Effects of Trehalose Injection in Unfed Females on Rhopr-TRET Transcript Expression in Fat Body and Ovaries}

The previous results suggest that $\mathrm{AKH}$ and insulin signaling could contribute substantially to trehalose homeostasis through the regulation of Rhopr-TRET transcript expression. We therefore questioned if injection of trehalose into unfed females could modify the transcripts involved in such signaling (Figure 8A). In our experimental condition, trehalose injection significantly increases Rhopr-TRET transcript expression in the fat body $(p=0.031$, by the Student's $t$-test, $n=5$ ) at $3 \mathrm{~h}$ post-injection, but not in the ovaries (Figures $\mathbf{8 B}, \mathbf{C}$ ). In addition, we examined transcripts involved with the insulin and $\mathrm{AKH}$ signaling pathway after trehalose injection. Figures $8 \mathrm{D}, \mathbf{F}$ show that following trehalose injection Rhopr-IGF and Rhopr-ILP1 transcript levels significantly increase in the fat body and CNS, respectively, demonstrating a potential stimulatory effect on insulin signaling, which is not observed in ovaries (Figure 8E). In contrast, Rhopr-AKH transcript expression tends to decrease in the CNS $(p>0.05$, by Student's $t$-test, $n=4$ ) (Figure $\mathbf{8 G}$ ). To confirm the possible regulation of Rhopr-TRET by insulin signaling, we down-regulated Rhopr-IR1 in unfed females (as described above) and 1 day after dsRNA treatment, we injected trehalose (Figure $\mathbf{8 H}$ ). Insects with down-regulated Rhopr-IR1 are not able to increase RhoprTRET expression in the fat body after trehalose injection (dsRhopr-IR1/T) to the same levels as those of control insects (dsARG/T) (Figures 8I,J). This finding shows that trehalose seems to be an important signal for controlling Rhopr-TRET mRNA levels via insulin signaling at least in the fat body.

Taking into account the premise that $\mathrm{AKH}$ signaling has been widely studied as a catabolic cascade involved in energy production under unfavorable conditions, we evaluated the abundance of transcripts involved in $\mathrm{AKH}$ signaling during the unfed state on the 3 key tissues implicated in reproductive performance. As can be seen in Figures 7A,B, expression of Rhopr-AKHR transcript in the fat body and ovaries increases as the unfed condition advances, with higher levels at 30 days post-ecdysis. Accordingly, the Rhopr-AKH transcript expression in $\mathrm{CNS}$ also increases at 30 days post-ecdysis (Figure 7C). Interestingly, the survival of unfed insects with knock down for Rhopr-AKHR significantly decreases with respect to controls (dsARG) (Figure 7D) 2 days post dsRNA treatment. Overall, these results suggest that $\mathrm{AKH}$ signaling could play a leading role during starvation.

\section{DISCUSSION}

Hematophagous insects, like triatomines, feed on blood that is rich in proteins but relatively poor in lipids and carbohydrates. However, the accumulation of carbohydrate 
by the eggs of $R$. prolixus is of some importance to support successful embryogenesis (Santos et al., 2008). Taking this into consideration, we show here the potential control by ILPs and $\mathrm{AKH}$ signaling cascades over trehalose uptake by ovaries. In addition, we suggest that Rhopr-TRET may work cooperatively with $\mathrm{AKH}$ signaling to support the release of trehalose from tissues into the hemolymph to be used as a source of fuel during stressful situations such as starvation.

Glycogen represents an important energy reserve in all animal cells, including the oocytes of insects (Arrese and Soulages, 2010), since glycogen, along with lipids, are primarily utilized in the metabolic production of ATP and carbon sources. While proteins

A Porcine insulin (P_In) or R. prolixus AKH (Rhopr-AKKH) treatment

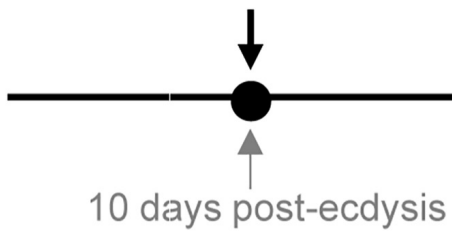

B

Rhopr-TRET

C

Ovaries collection
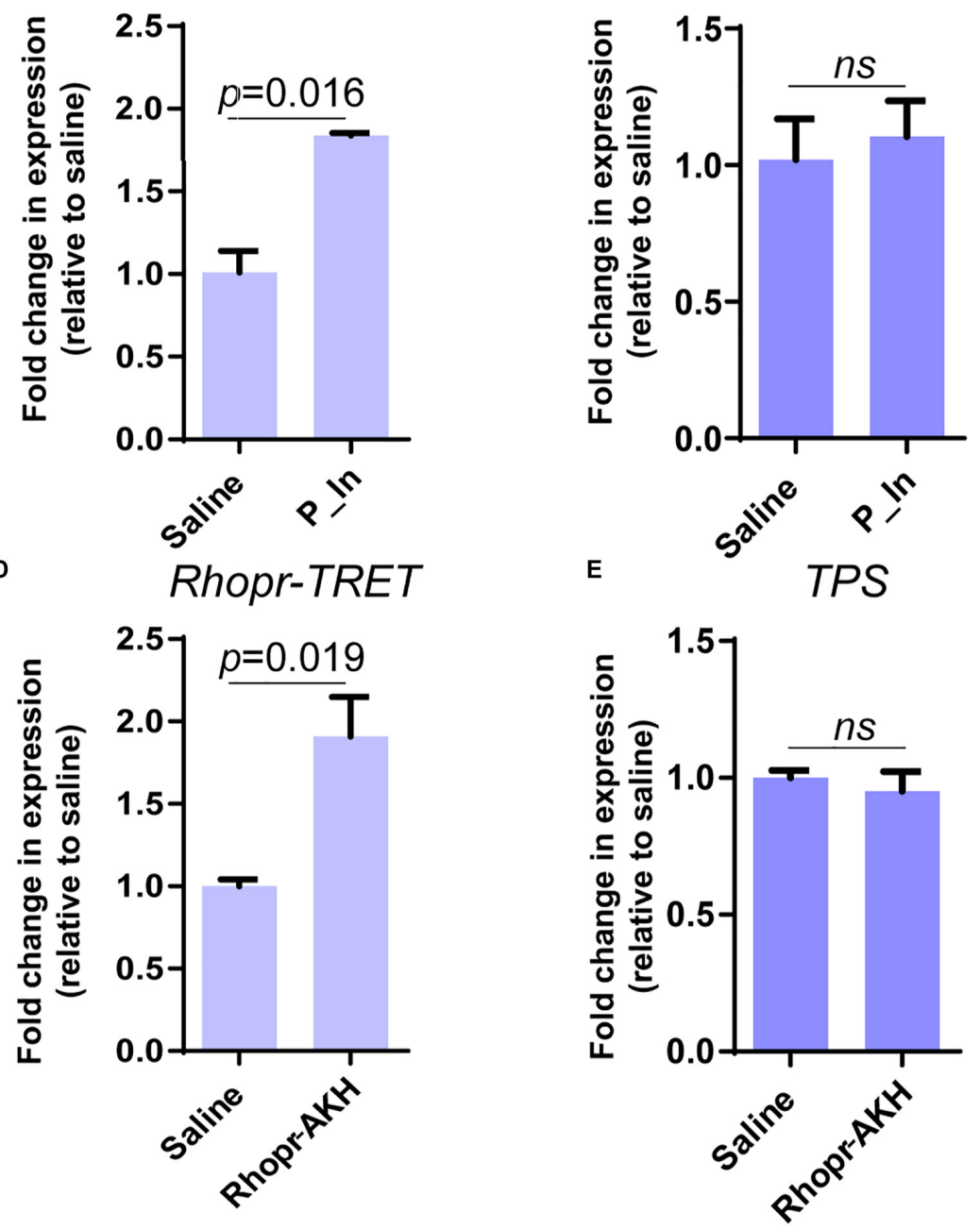

FIGURE 5 | Participation of insulin and AKH signaling in Rhopr-TRET mRNA expression using in vivo assays. (A) Experimental scheme for (B-E). (B) Rhopr-TRET transcript expression after P_In injection; (C) TPS transcript expression after P_In injection; (D) Rhopr-TRET transcript expression after Rhopr-AKH injection. (E) TPS transcript expression after Rhopr-AKH injection. Rhopr-TRET and TPS transcript expression in ovaries of unfed adult females was tested by RT-qPCR. The $y$-axis represents the fold change in expression relative to control (saline, value $\sim 1)$. The results are shown as the mean $\pm \operatorname{SEM}(n=3-4$, where each $n$ represents a pool of tissues from 2 insects). Statistically significant differences were determined by the Student's $t$-test. 


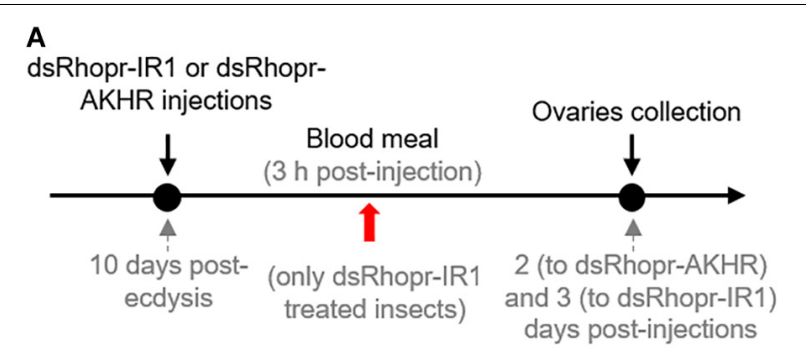

B
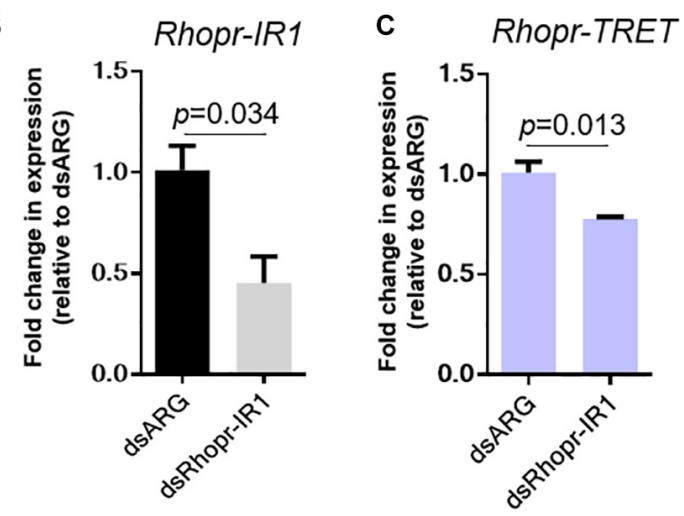

D
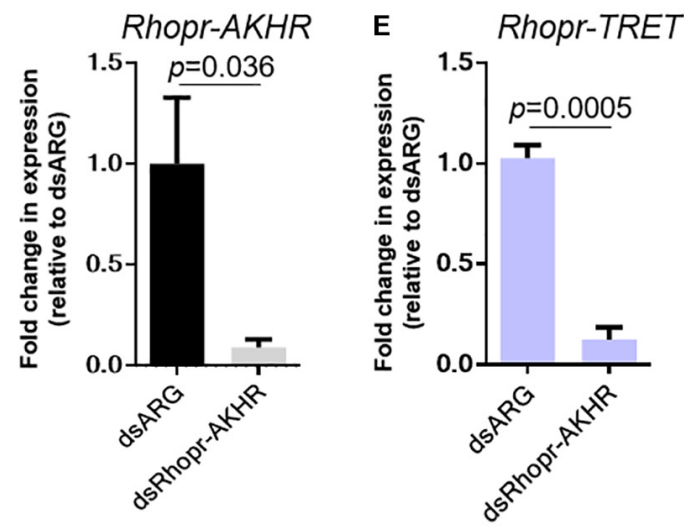

F dsRhopr-IR1 or dsRhopr-AKHR

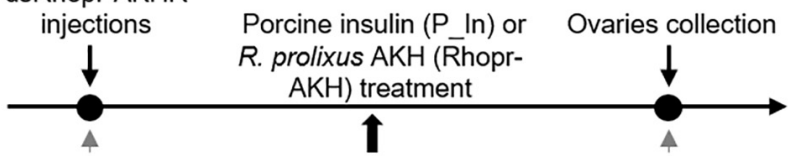

10 days postecdysis
1 day post-

injection
$3 \mathrm{~h}$ posttreatment
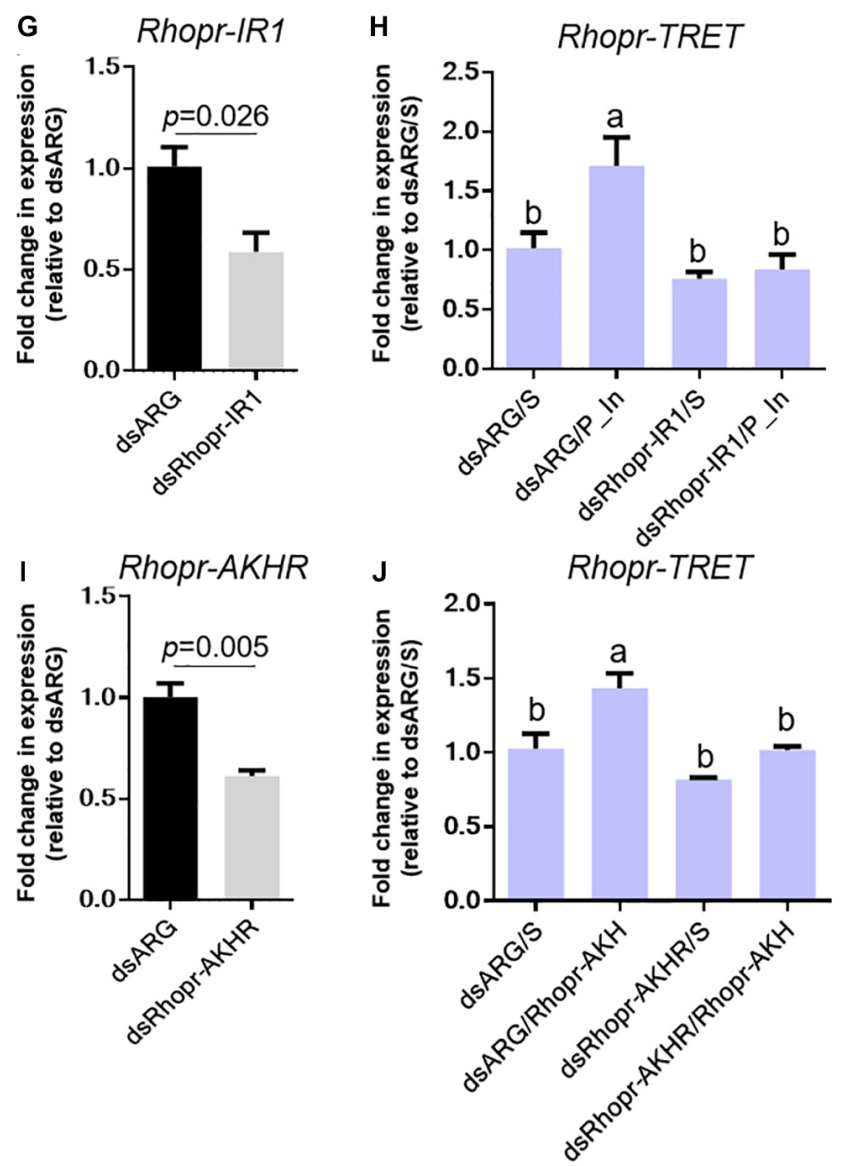

FIGURE 6 | Effect of dsRNA treatment on Rhopr-TRET mRNA expression. (A) Experimental scheme for (B-E). Panels (B,D) show the knockdown efficiency of dsRhopr-IR1 and dsRhopr-AKHR treatments, respectively.Panels (C,E) show the effects of down-regulation of Rhopr-IR1 and Rhopr-AKHR transcripts on Rhopr-TRET expression. (F) Experimental scheme for (G-J). Panels (G,I) show the knockdown efficiency of dsRhopr-IR1 and dsRhopr-AKHR treatments, respectively. Panels $\mathbf{( H , J )}$ show the effects of down-regulation of Rhopr-IR1 and Rhopr-AKHR transcripts on Rhopr-TRET mRNA expression following P_In (H), Rhopr-AKH (J) or saline stimulation. Transcript expression was tested by RT-qPCR. The $y$-axis represents the fold change in expression relative to dsARG injection after saline treatment (dsARG/S, value $\sim 1$ ) obtained viageometric averaging using $18 \mathrm{~S}$ ribosomal RNA subunit and $\beta$-actin as reference genes. The results are shown as the mean \pm SEM $(n=4-5$, where each $n$ represents an individual tissue from 1 insect). Statistically significant differences were determined by Student's $t$-test in all graphs except for $\mathbf{( H , J )}$ in which a one-way ANOVA and Tukey's test as post hoc test was used (different letters indicate significant difference at $P<0.05)$. dsARG/S: dsARG insects with saline injection; dsARG/P_In: dsARG insects with porcine insulin injection: dsARG/Rhopr-AKH: dsARG insects with Rhopr-AKH injection; dsRhopr-IR1/S: dsRhopr/IR1 insects with saline injection; dsRhopr-IR1/P_In: dsRhopr-IR1 insects with porcine insulin injection; dsRhopr-AKHR/S: dsRhopr-AKHR insects with saline injection; dsRhopr-AKHR/Rhopr-AKH: dsRhopr-AKHR insects with Rhopr-AKH injection.

and lipids are sequestered by the developing oocyte from extraovarian tissues (Raikhel and Dhadialla, 1992; Canavoso et al., 2001), glycogen is synthesized in the ovary itself. The fat body is the major site of synthesis of trehalose in insects, with the trehalose capable of being released into the circulation for use by other tissues, including ovaries, depending on the specific physiological state (Tang et al., 2018). Here, we show that before a blood meal, the amount of trehalose in the fat body and ovaries is very low or not detectable. This is to be expected since trehalose is not used for carbohydrate accumulation, but rather as an intermediary between what is stored as glycogen and what is transferred to other tissues. Unfed or starved insects tend to 


\section{AKH signaling during the unfed condition}

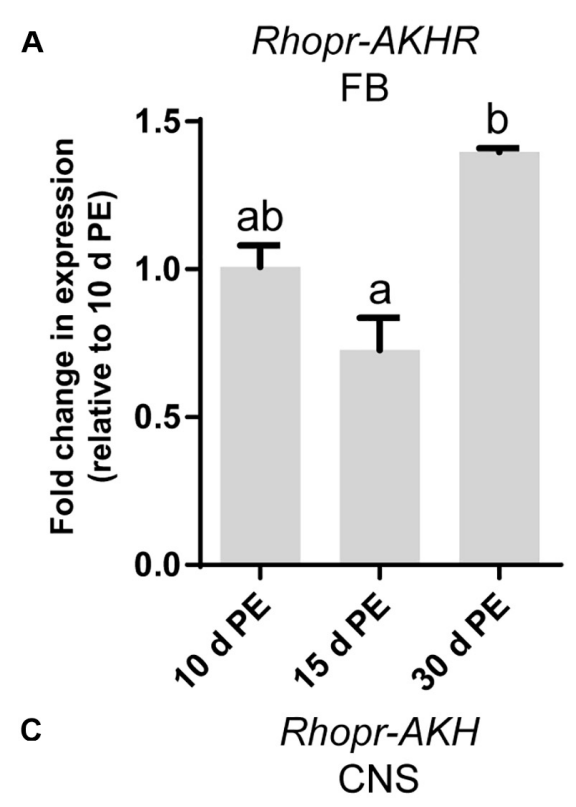

B
OV
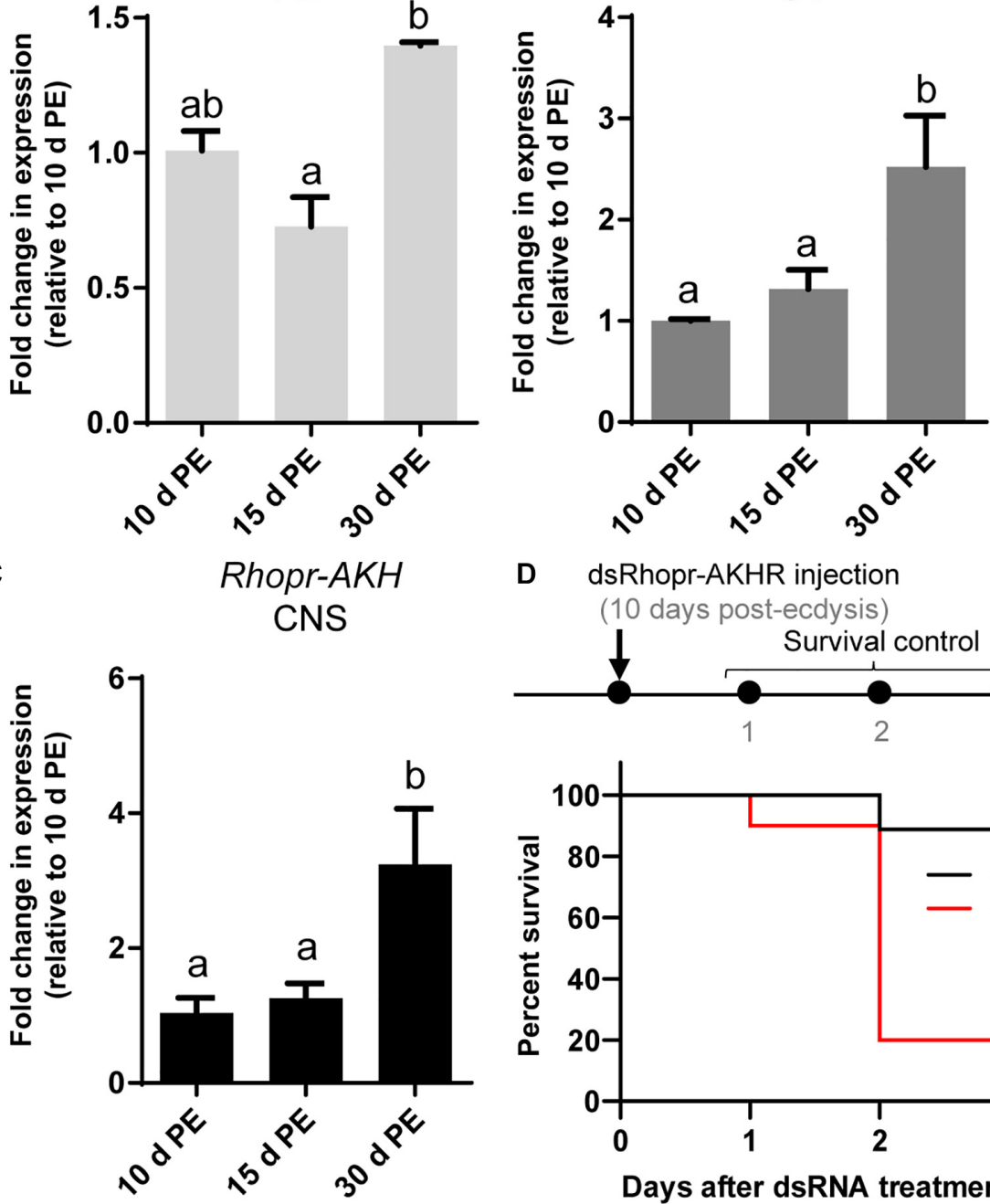

D dsRhopr-AKHR injection
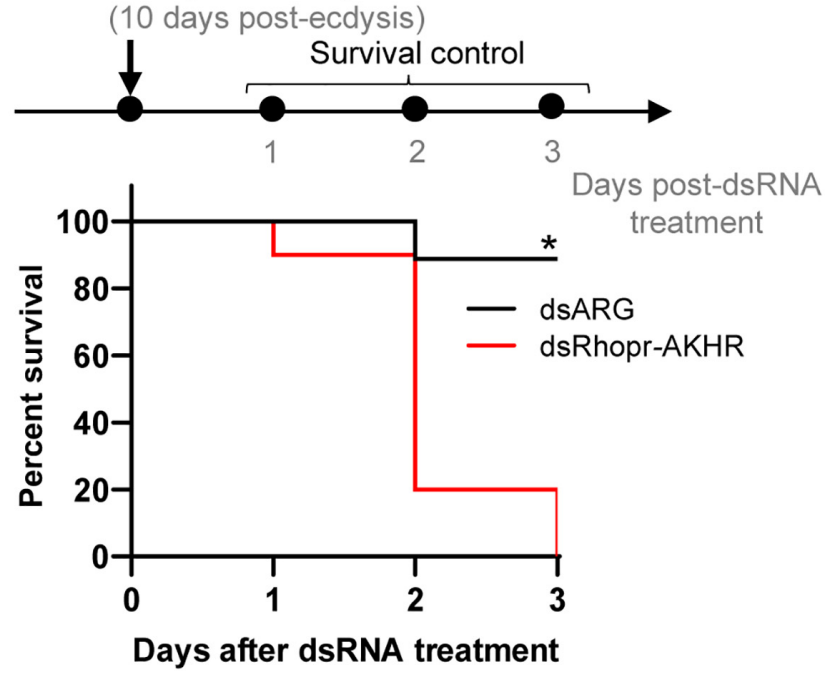

FIGURE 7 | Transcript expression of Rhopr-AKHR and Rhopr-AKH in fat body, ovaries and CNS following ecdysis into adults. Rhopr-AKHR transcript expression in the fat body (A) and ovaries (B), and Rhopr-AKH transcript expression in the CNS (C) were assessed at various times post-ecdysis (PE) in adult females. The effects were measured by RT-qPCR. The $y$-axis represents the fold change in expression relative to 10 days PE (value $\sim 1$ ). The results are shown as the mean \pm SEM $(n=3-4$, where each $n$ represents a pool of tissues from 3 insects). The statistically significant differences were determined by a one-way ANOVA and a Tukey's test as the post hoc test. Significance of $P<0.05$ is denoted using letters to indicate bars that are significantly different from others. (D) Percent survival of dsRhopr-AKHR injected insects with respect to control (dsARG) over 3 days in the unfed condition $(n=15)$. Differences in the survival curve were analyzed using the Log-Rank test ( $\left.{ }^{*} p<0.001\right)$.

break down nutrients stored in tissues and then release them into the hemolymph to sustain the female's lifespan until nutritional shortage is alleviated (Bell and Bohm, 1975; Leyria et al., 2014). In $R$. prolixus, the glycogen stored in the ovaries or fat body during the unfed condition (Leyria et al., 2020b) may progressively decline due to trehalose synthesis and release into the circulation for use by other tissues; indeed, trehalose is detected in the fat body of $R$. prolixus females under long periods of starvation. This may be the reason why there are measurable levels of circulating trehalose during the unfed condition. In a related triatomine species, Dipetalogaster maxima, ovarian nutritional resources, including carbohydrates, decrease when nutritional resources are scarce, reflecting adjustments in metabolism due to the physiological needs of the female (Leyria et al., 2014).

In $R$. prolixus females, a blood meal triggers an increase in trehalose concentration in the fat body, hemolymph and ovaries, reaching maximum at 4-5 days after feeding. In our experimental conditions, $R$. prolixus females begin laying eggs 5 days after a blood meal (Leyria et al., 2020a) and due to the asynchronous development of the ovarioles (Atella et al., 2005; 


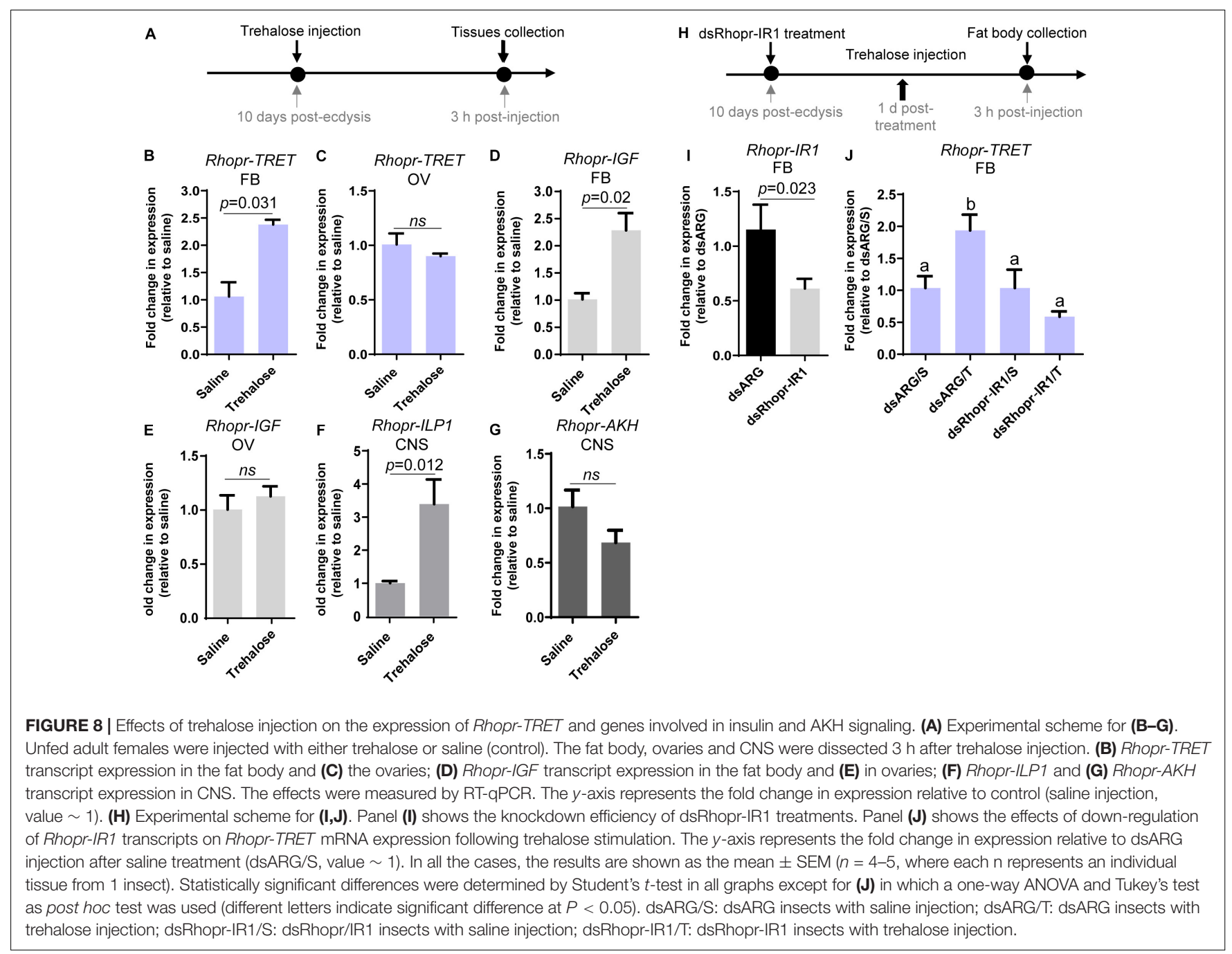

Aguirre et al., 2011), triatomine females can lay eggs for at least 25-30 days. During this period, carbohydrates should be constantly produced, mainly by the key tissue involved in nutrient metabolism, the fat body, released and then stored by the oocytes. We evaluated the dynamics of the enzymes involved in trehalose homeostasis and found that in ovarian tissue, TPS remains almost constant during all time points evaluated in both nutritional states; however, in the fat body, TPS is upregulated by $\sim$ four-sixfold after a blood meal. Identifying that trehalose increases in the ovary after a blood meal is the first indication that its synthesis by the fat body may be necessary for the accumulation of carbohydrates by the ovaries during this rapid developmental phase of the oocytes, i.e., vitellogenesis (Valle, 1993; Roy et al., 2018). TPS has previously been detected and/or quantified in fat bodies of several species (Cui and Xia, 2009; Xu et al., 2009; Chen et al., 2010; Tang et al., 2010, 2018; Xiong et al., 2019; Liu et al., 2020) including $R$. prolixus females, where a transcriptome analysis reveals an up-regulation of this enzyme in the fat body of fed females (Leyria et al., 2020b). Interestingly, in $R$. prolixus, the concentration of trehalose in the fat body and hemolymph is lower than that found in other non-hematophagous species (Oda et al., 2000; Singtripop et al., 2002; Moriwaki et al., 2003; Michitsch and Steele, 2008; Huang et al., 2012; Kim and Hong, 2015; Kh and Keshan, 2019) and, more importantly, it is also lower compared to that found in $R$. prolixus males (Mariano et al., 2009). There is a peak of trehalose in the fat body of females around 5 days postblood meal ( $600 \mathrm{pmol} /$ fat body), while in males the maximum trehalose concentration is shown around 5-6 days post-feeding, but in the range of 30-40 nmol/tissue (Mariano et al., 2009). However, although trehalose titers in hemolymph of adult males is slightly higher at 3-4 days post-blood meal, the titers are similar to those found in females. The low amount of trehalose in $R$. prolixus compared to other insects may be due to its diet being carbohydrate poor. Overall, the results above indicate that in $R$. prolixus, carbohydrate metabolism is, in part, determined by sex, even at the same stage of development and nutritional state.

It is well known that mobilization of trehalose is critical for metabolic homeostasis (Steele, 1981; Arrese and Soulages, 2010). Trehalase enzymes and TRET are essential for this mobilization, with trehalase responsible for the breakdown of trehalose to glucose (Shukla et al., 2015), and TRET required 
to transfer trehalose via the hemolymph from one tissue to another (Kanamori et al., 2010). As expected, trehalase mRNA levels, mainly the soluble form (s-Tre), are up-regulated during the unfed condition in both the fat body and ovaries of $R$. prolixus females. This finding further supports the hypothesis that nutritional shortage promotes not only the transfer of nutritional sources to other tissues, but also trehalose degradation (in situ) for energy generation. Although in some insects, such as the beet armyworm Spodoptera exigua, starvation induces an increase in circulating trehalose titers (Kim and Hong, 2015), in our experimental conditions the concentration of trehalose in the hemolymph of unfed $R$. prolixus females is the lowest of all the time points analyzed and this was also observed in unfed males by Mariano et al. (2009).

TRET was first described in the anhydrobiotic insect, Polypedilum vanderplanki and now known to be involved in the permeability of cells to trehalose in invertebrates and vertebrates (Kikawada et al., 2007). Rhopr-TRET is part of a mono-clade among the insect sugar transporters. The amino acid sequence of Rhopr-TRET is similar to transporters found to facilitative trehalose transport, particularly those found in other hemipterans, such as C. lectularius and $H$. halys. Interestingly, in N. lugens, a specific TRET, named Nlst8, is principally expressed in Malpighian tubules and is involved in trehalose reabsorption (Kikuta et al., 2012). Another disaccharide transporter, SCRT, was identified in Drosophila melanogaster, and is mainly involved in sucrose uptake in the intestinal tract, especially the hindgut (Meyer et al., 2011). In $R$. prolixus the highest expression of Rhopr-TRET is found in the fat body. Interestingly, we also show that Rhopr-TRET is upregulated in the fat body after feeding, which is further supported by a recently published transcriptome analysis (Leyria et al., 2020b). This up-regulation remains throughout all the time points analyzed during the fed state, suggesting that the trehalose synthesized in the fat body is transported via Rhopr-TRET into the hemolymph. The circulating trehalose levels stay relatively stable until day 4 post-blood meal, but on day 5 increase three-fourfold. However, trehalose contained in the ovaries begins to be detected after day 1 post-blood meal. Despite this fact, TPS transcript levels in the ovaries do not show any obvious differences after feeding, indicating the importance of trehalose uptake by the ovaries rather than its synthesis. Santos et al. (2008, 2012) reported that follicles containing vitellogenic oocytes have higher trehalase activity, mainly due to a membrane-bound trehalase (m-Tre), which may provide glucose for carbohydrate accumulation, as demonstrated in Bombyx mori ( $\mathrm{Su}$ et al., 1994). Interestingly, in $R$. prolixus, Rhopr-TRET is up-regulated after feeding $(\sim 7-$ 8 fold), coincident with the increase in trehalose in the ovaries, indicating that this carbohydrate could be transported from the hemolymph via Rhopr-TRET into the oocytes. These findings are not contradictory with those reported by Santos et al. (2008, 2012), but rather suggest that redundant pathways are synchronized for the same purpose in order to maximize the storage of carbohydrate by oocytes in a short period of time, as was demonstrate for lipid accumulation in other related species (Fruttero et al., 2011; Leyria et al., 2014).
Thus, although Rhopr-TRET allows the release of trehalose from the fat body, it also seems to facilitate trehalose uptake by ovaries.

The responses of Rhopr-TRET may depend not only on the tissue in which it is expressed but also on specific hormonal control. As in mammals, circulating sugar levels in insects are mainly regulated by the action of two peptide hormone families, ILPs and AKHs (Toprak, 2020). The control of trehalose metabolism by ILPs, including circulating trehalose levels, as well as their participation in regulating the expression of trehalases and TPS, has been reported in several species (Bounias et al., 1993; Satake et al., 1997; Iwami, 2000; Wu and Brown, 2006; Broughton et al., 2008; Wang et al., 2020). However, until now, the link between insulin signaling and TRET has been scarcely studied, even though in vertebrates glucose transporter 4 (GLUT4) is translocated to the plasma membrane in response to insulin signals (Bryant and Gould, 2020). In this study, the down-regulation of Rhopr-IR1 through RNA-mediated interference, and stimulation with exogenous insulins, show that insulin signaling is positively influencing Rhopr-TRET transcript expression in the ovaries of fed insects, a state where anabolic processes are required for egg formation and where it is known that the insulin signaling cascade is activated (Leyria et al., 2020a). Interestingly, this regulation is specific to Rhopr-TRET and not TPS, suggesting that insulin signaling is promoting trehalose mobilization more than its synthesis. When trehalose is injected into unfed females, mimicking a "fed condition," Rhopr-ILP1 transcript expression increases in the CNS, and Rhopr-IGF and Rhopr-TRET transcripts increase in the fat body, demonstrating the importance of insulin cascade activation when the organism detects sources to promote anabolic processes. These effects following trehalose injection are not observed in the ovaries, showing the importance of the fat body as the main modulator of metabolic processes.

The down-regulation of Rhopr-AKHR through RNA-mediated interference, as well as the stimulation with exogenous Rhopr$\mathrm{AKH}$, reveals that the Rhopr-AKH signaling pathway is positively modulating the expression of Rhopr-TRET mRNA in ovaries of $R$. prolixus females but no significant differences were observed in the expression of TPS. A comparable result was recently reported in Locusta migratoria, where down-regulation of $A K H R$ in the fat body decreases the expression of TRET but not of TPS (Zheng et al., 2020). We do not know how the nutritional state might be influencing this control. The first indication of the now named $\mathrm{AKH} /$ Red pigment-concentrating hormones $(\mathrm{AKH} / \mathrm{RPCH})$ family in insects was that of a hyperglycemic factor in the American cockroach Periplaneta americana (Steele, 1961). AKHs work by mobilizing energy reserves in the fat body and simultaneously inhibiting the storage of proteins, lipids or glycogen during periods of stressful situations, such as starvation (Lorenz and Gäde, 2009). However, studies using the cricket, Gryllus bimaculatus, have proposed that $\mathrm{AKH}$ could trigger the mobilization of energy substrates that are incorporated into growing oocytes (Lorenz and Anand, 2004; Lorenz and Gäde, 2009). In the nematode Caenorhabditis elegans, an AKH-like peptide was also reported as positively influencing reproductive processes (Lindemans et al., 2009) and in the tsetse 
fly Glossina morsitans and the oriental fruit fly Bactrocera dorsalis, down-regulation of AKHR leads to a significant reduction of fecundity (Attardo et al., 2012; Hou et al., 2017). In addition, in N. lugens, AKH/AKHR signaling-mediated maintenance of trehalose levels in the hemolymph is closely associated with vitellogenin uptake and maturation of oocytes (Lu et al., 2019). Thus, understanding that AKH activates glycogen phosphorylase, an enzyme that leads to decreased glycogen stores and a subsequent increase in the production of trehalose (Gäde et al., 1997), we hypothesize that during vitellogenesis, $R$. prolixus females might require the mobilization of trehalose as well as other nutrients, via AKH signaling. Moreover, we cannot rule out that this signaling is also stimulating the expression of RhoprTRET in the ovaries thereby maximizing trehalose uptake during egg formation. Interestingly, it was recently reported that $\mathrm{JH}$, a key hormone for triggering vitellogenesis in triatomines, induces transcript expression of TRET in the ovaries of the cabbage beetle Colaphellus bowringi, encouraging further studies on the interplay of different hormonal pathway in the regulation of TRET (Li et al., 2020).

Rhopr-AKHR transcript levels in the fat body and ovaries, along with the Rhopr-AKH transcript in the CNS, increase as the unfed condition progresses. Most importantly, the survival of
dsRhopr-AKHR treated females decreases, indicating how vital $\mathrm{AKH}$ signaling is during a nutritional shortage. In $R$. prolixus, AKHR is regulated at the transcriptional level and is required for lipid mobilization under starvation (Alves-Bezerra et al., 2016). In starved insects of $S$. exigua, high titers of trehalose in the hemolymph arise from its release from tissues through TRET (Tang et al., 2012; Kim and Hong, 2015). Similarly, in the red flour beetle Tribolium castaneum, TRET transcript levels are higher in starved beetles and regulated by $\mathrm{JH}$ and not insulin signaling (Xu et al., 2013). These finding indicates that during the unfed condition Rhopr-TRET may be controlled by AKH signaling to mobilize carbohydrates. Hypertrehalosemic hormone (HrTH) belongs to the AKH/RPCH family (Keeley and Gäde, 2004). In a cockroach, Blattella germanica, HrTH is released during starvation to control the expression of genes related to catabolic processes via the FoxO (Forkhead box O) transcriptional factor (Süren-Castillo et al., 2014). Recently, we suggested that FoxO signaling is activated in ovaries and fat bodies of unfed females via down-regulation of insulin signaling (Leyria et al., 2020a). Considering this, the fine control of trehalose homeostasis via Rhopr-TRET during the unfed condition may be explained by the result of two antagonistic hormonal actions; the inhibition of insulin signaling and the up-regulation of $\mathrm{AKH}$ signaling.

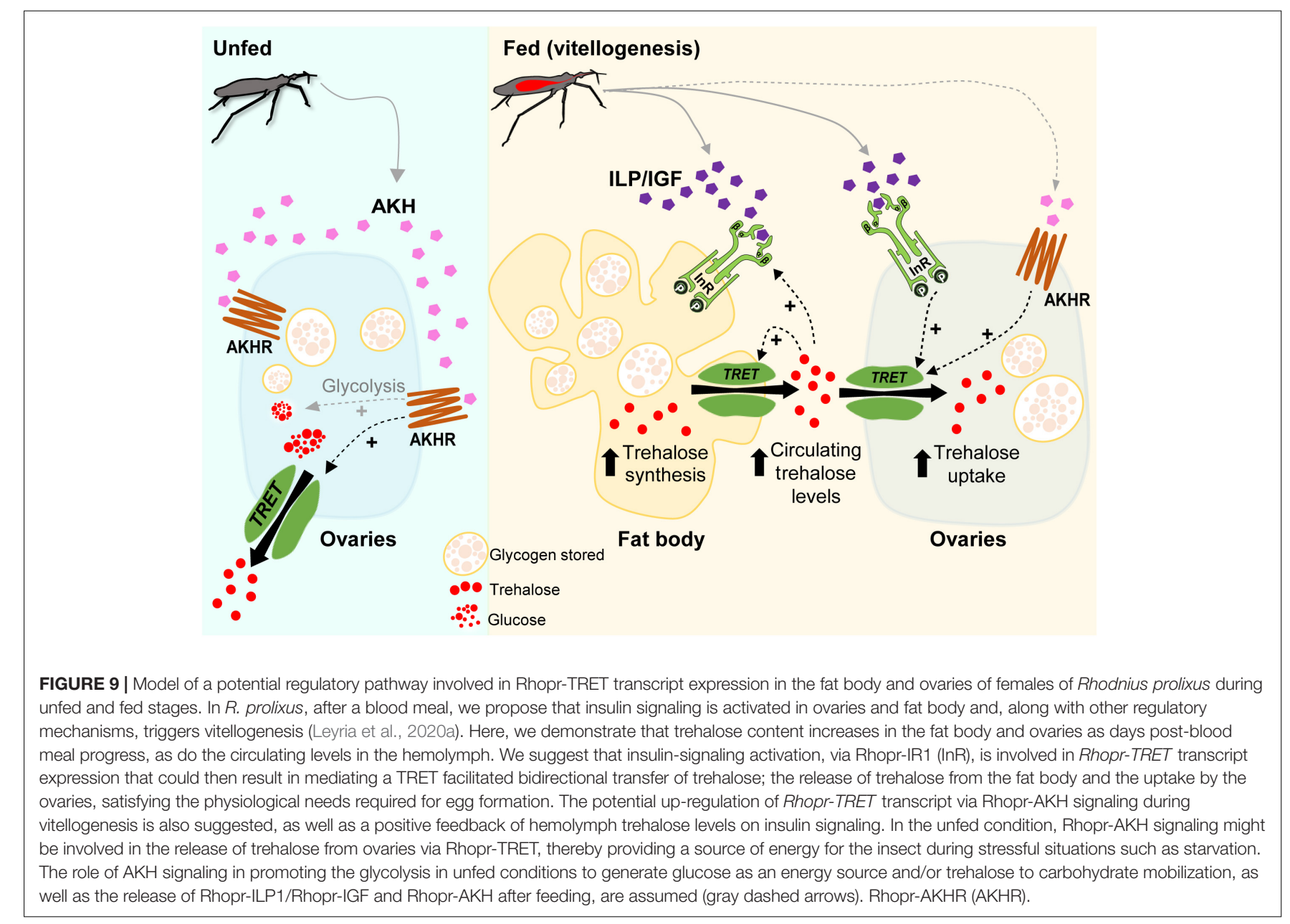


We should point out that treatment with exogenous trehalose in unfed females promotes a decrease in the Rhopr-AKH transcript in the CNS, suggesting that $\mathrm{AKH}$ synthesis is down-regulated under nutrient stimulation. Coincidently, in L. migratoria, by in vitro assay, it was reported that exogenous trehalose inhibits the release of AKHs from the CC, at the level of the adipokinetic hormone-producing cells (Passier et al., 1997). Furthermore, when we evaluated the effects on Rhopr-TRET transcript expression in the ovaries by injection of hormones (ex vivo assays), Rhopr-AKH (3 h) appears to act faster than insulins (12 h), suggesting that the ovaries are more responsive to $\mathrm{AKH}$ than to insulin in an unfed state. Also, we evaluated the expression of the TPS transcript after manipulations to down- or up- regulate $\mathrm{AKH}$ and insulin signaling and the results show no significant differences compared to control insects. The same effect was observed in the beet armyworm S. exigua (Park and Kim, 2017).

Although the effects of insulin and $\mathrm{AKH}$ signaling on nutrient mobilization have been reported in $R$. prolixus (Marco et al., 2013; Patel et al., 2014; Zandawala et al., 2015; Alves-Bezerra et al., 2016; Defferrari et al., 2016a,b), the potential molecular mechanisms by which these hormones act on trehalose homeostasis have not previously been studied. Here, we demonstrate that trehalose content increases in the fat body and ovaries as days-post-blood meal progress, as do the circulating levels of trehalose in the hemolymph. We suggest that insulin-signaling activation, via Rhopr-IR1, is involved in Rhopr-TRET transcript expression that could then result in mediating a TRET facilitated bidirectional transfer of trehalose: the release of trehalose from the fat body and the uptake by the ovaries, satisfying the physiological needs required for egg formation. The potential up-regulation of Rhopr-TRET transcript via Rhopr-AKH signaling during vitellogenesis is also suggested, as well as a positive feedback of hemolymph trehalose levels on insulin signaling. In the unfed condition, Rhopr-AKH signaling might be involved in the release of trehalose from the ovaries via Rhopr-TRET, thereby providing a source of energy for the insect during stressful situations such as starvation (Figure 9) although the transcript

\section{REFERENCES}

Aguirre, S. A., Fruttero, L. L., Leyria, J., Defferrari, M. S., Pinto, P. M., Settembrini, B. P., et al. (2011). Biochemical changes in the transition from vitellogenesis to follicular atresia in the hematophagous Dipetalogaster maxima (Hemiptera: Reduviidae). Insect Biochem. Mol. Biol. 41, 832-841. doi: 10.1016/j.ibmb.2011. 06.005

Alves-Bezerra, M., De Paula, I. F., Medina, J. M., Silva-Oliveira, G., Medeiros, J. S., Gäde, G., et al. (2016). Adipokinetic hormone receptor gene identification and its role in triacylglycerol metabolism in the blood-sucking insect Rhodnius prolixus. Insect Biochem. Mol. Biol. 69, 51-60. doi: 10.1016/j.ibmb.2015. 06.013

Arrese, E. L., and Soulages, J. L. (2010). Insect fat body: energy, metabolism, and regulation. Annu. Rev. Entomol. 55, 207-225. doi: 10.1146/annurev-ento112408-085356

Artimo, P., Jonnalagedda, M., Arnold, K., Baratin, D., Csardi, G., de Castro, E., et al. (2012). ExPASy: SIB bioinformatics resource portal. Nucleic Acids Res. 40, W597-W603. doi: 10.1093/nar/gks400

Atella, G. C., Gondim, K. C., Machado, E. A., Medeiros, M. N., Silva-Neto, M. A., and Masuda, H. (2005). Oogenesis and egg development in triatomines: a expression of Rhopr-TRET is low in unfed insects relative to fed insects. The results indicate that in females of $R$. prolixus, trehalose homeostasis and its hormonal regulation by insulin and $\mathrm{AKH}$ signaling could play critical roles in adapting to different nutritional conditions. Further experiments using biochemical or functional assays could possibility reveal the entire regulatory mechanism of the trehalose metabolism in triatomines.

\section{DATA AVAILABILITY STATEMENT}

The original contributions generated for this study are included in the article/Supplementary Material, further inquiries can be directed to the corresponding author.

\section{AUTHOR CONTRIBUTIONS}

JL, AL, and IO designed the experiments and mapped out the manuscript. JL and HE-M performed the experiments. JL wrote the original draft of the manuscript and prepared all the figures. $\mathrm{AL}$ and $\mathrm{IO}$ edited, reviewed, and contributed to the writing of the manuscript. All authors contributed to the article and approved the submitted version.

\section{FUNDING}

This study was supported by the Natural Sciences and Engineering Research Council of Canada Discovery grants to AL (RGPIN-2019-05775) and IO (RGPIN-2017-06402).

\section{SUPPLEMENTARY MATERIAL}

The Supplementary Material for this article can be found online at: https://www.frontiersin.org/articles/10.3389/fphys. 2021.624165/full\#supplementary-material

biochemical approach. An. Acad. Bras. Cienc. 77, 405-430. doi: 10.1590/s00017652005000300005

Attardo, G. M., Benoit, J. B., Michalkova, V., Yang, G., Roller, L., Bohova, J., et al. (2012). Analysis of lipolysis underlying lactation in the tsetse fly, Glossina morsitans. Insect Biochem. Mol. Biol. 42, 360-370. doi: 10.1016/j.ibmb.2012. 01.007

Badisco, L., Van Wielendaele, P., and Vanden Broeck, J. (2013). Eat to reproduce: a key role for the insulin signalling pathway in adult insects. Front. Physiol. 4:202. doi: 10.3389/fphys.2013.00202

Bednáøová, A., Kodrík, D., and Krishnan, N. (2013). Unique roles of glucagon and glucagon-like peptides: parallels in understanding the functions of adipokinetic hormones in stress responses in insects. Comp. Biochem. Physiol. A Mol. Integr. Physiol. 164, 91-100. doi: 10.1016/j.cbpa.2012.10.012

Bell, W. J., and Bohm, M. K. (1975). Oosorption in insects. Biol. Rev. Camb. Philos. Soc. 50, 373-396. doi: 10.1111/j.1469-185x.1975.tb01058.x

Blom, N., Gammeltoft, S., and Brunak, S. (1999). Sequence and structure-based prediction of eukaryotic protein phosphorylation sites. J. Mol. Biol. 294, 13511362. doi: 10.1006/jmbi.1999.3310

Bounias, M., Bahjou, A., Gourdoux, L., and Moreau, R. (1993). Molecular activation of a trehalase purified from the fat body of a coleopteran insect 
(Tenebrio molitor), by an endogenous insulin-like peptide. Biochem. Mol. Biol. Int. 31, 249-266.

Brosnan, J. T. (1999). Comments on metabolic needs for glucose and the role of gluconeogenesis. Eur. J. Clin. Nutr. 53, S107-S111. doi: 10.1038/sj.ejcn.1600748

Broughton, S., Akic, N., Slack, C., Bass, T., Ikeya, T., Vinti, G., et al. (2008). Reduction of DILP2 in Drosophila triages a metabolic phenotype from lifespan revealing redundancy and compensation among DILPs. PLoS One 3:e3721. doi: 10.1371/journal.pone.0003721

Bryant, N. J., and Gould, G. W. (2020). Insulin stimulated GLUT4 translocation Size is not everything! Curr. Opin. Cell Biol. 65, 28-34. doi: 10.1016/j.ceb.2020. 02.006

Canavoso, L. E., Jouni, Z. E., Karnas, K. J., Pennington, J. E., and Wells, M. A. (2001). Fat metabolism in insects. Annu. Rev. Nutr. 21, 23-46. doi: 10.1146/ annurev.nutr.21.1.23

Chen, J., Zhang, D., Yao, Q., Zhang, J., Dong, X., Tian, H., et al. (2010). Feedingbased RNA interference of a trehalose phosphate synthase gene in the brown planthopper, Nilaparvata lugens. Insect Mol. Biol. 19, 777-786. doi: 10.1111/j. 1365-2583.2010.01038.x

Cruz, J., Mané-Padrós, D., Bellés, X., and Martín, D. (2006). Functions of the ecdysone receptor isoform-A in the hemimetabolous insect Blattella germanica revealed by systemic RNAi in vivo. Dev. Biol. 297, 158-171. doi: 10.1016/j.ydbio. 2006.06.048

Cui, S. Y., and Xia, Y. X. (2009). Isolation and characterization of the trehalose6-phosphate synthase gene from Locusta migratoria manilensis. Insect Sci. 16, 287-295. doi: 10.1111/j.1744-7917.2009.01268.x

de Azambuja, P., Garcia, E. S., and Ratcliffe, N. A. (1991). Aspects of classification of Hemiptera hemocytes from six triatomine species. Mem. Inst. Oswaldo Cruz. 86, 1-10. doi: 10.1590/s0074-02761991000100002

Defferrari, M. S., Da Silva, S. R., Orchard, I., and Lange, A. B. (2018). A Rhodnius prolixus insulin receptor and its conserved intracellular signalling pathway and regulation of metabolism. Front. Endocrinol. 9:745.

Defferrari, M. S., Orchard, I., and Lange, A. B. (2016a). An insulin-like growth factor in Rhodnius prolixus is involved in post-feeding nutrient balance and growth. Front. Neurosci. 10:566. doi: 10.3389/fendo.2018. 00745

Defferrari, M. S., Orchard, I., and Lange, A. B. (2016b). Identification of the first insulin-like peptide in the disease vector Rhodnius prolixus: involvement in metabolic homeostasis of lipids and carbohydrates. Insect. Biochem. Mol. Biol. 70, 148-159. doi: 10.1016/j.ibmb.2015.12.009

Eberhard, F. E., Cunze, S., Kochmann, J., and Klimpel, S. (2020). Modelling the climatic suitability of Chagas disease vectors on a global scale. eLife 9:e52072. doi: 10.7554/eLife.52072

Elbein, A. D., Pan, Y. T., Pastuszak, I., and Carroll, D. (2003). New insights on trehalose: a multifunctional molecule. Glycobiology 13, 17R-27R. doi: 10.1093/ glycob/cwg047

El-Gebali, S., Mistry, J., Bateman, A., Eddy, S. R., Luciani, A., Potter, S. C., et al. (2019). The Pfam protein families database in 2019. Nucleic Acids Res. 47, D427-D432. doi: 10.1093/nar/gky995

Felsenstein, J. (1985). Confidence limits on phylogenies: an approach using the bootstrap. Evolution 39, 783-791. doi: 10.1111/j.1558-5646.1985.tb00420.x

Foster, W. A. (1995). Mosquito sugar feeding and reproductive energetics. Annu. Rev. Entomol. 40, 443-474. doi: 10.1146/annurev.en.40.010195.002303

Fruttero, L. L., Frede, S., Rubiolo, E. R., and Canavoso, L. E. (2011). The storage of nutritional resources during vitellogenesis of Panstrogylus megistus (Hemiptera: Reduviidae): the pathways of lipophorin in lipid delivery to developing oocytes. J. Insect Physiol. 57, 475-486. doi: 10.1016/j.jinsphys.2011.01.009

Gäde, G., Hoffmann, K. H., and Spring, J. H. (1997). Hormonal regulation in insects: facts, gaps, and future directions. Physiol. Rev. 77, 963-1032. doi: 10. 1152/physrev.1997.77.4.963

Hamoudi, Z., Lange, A. B., and Orchard, I. (2016). Identification and characterization of the corazonin receptor and possible physiological roles of the corazonin-signaling pathway in Rhodnius prolixus. Front. Neurosci. 10:357. doi: $10.3389 /$ fnins. 2016.00357

Hou, Q. L., Chen, E. H., Jiang, H. B., Wei, D. D., Gui, S. H., Wang, J. J., et al. (2017). Adipokinetic hormone receptor gene identification and its role in triacylglycerol mobilization and sexual behavior in the oriental fruit fly (Bactrocera dorsalis). Insect Biochem. Mol. Biol. 90, 1-13. doi: 10.1016/j.ibmb. 2017.09.006
Huang, J. H., Bellés, X., and Lee, H. J. (2012). Functional characterization of hypertrehalosemic hormone receptor in relation to hemolymph trehalose and to oxidative stress in the cockroach Blattella germanica. Front. Endocrinol. 2:114. doi: $10.3389 /$ fendo.2011.00114

Huang, J. H., and Lee, H. J. (2011). RNA interference unveils functions of the hypertrehalosemic hormone on cyclic fluctuation of hemolymph trehalose and oviposition in the virgin female Blattella germanica. J. Insect Physiol. 57, 858-864. doi: 10.1016/j.jinsphys.2011.03.012

Iwami, M. (2000). Bombyxin: an insect brain peptide that belongs to the insulin family. Zool. Sci. 17, 1035-1044. doi: 10.2108/zsj.17.1035

Jones, D. T., Taylor, W. R., and Thornton, J. M. (1992). The rapid generation of mutation data matrices from protein sequences. Comput. Appl. Biosci. 8, 275-282. doi: 10.1093/bioinformatics/8.3.275

Kanamori, Y., Saito, A., Hagiwara-Komoda, Y., Tanaka, D., Mitsumasu, K., Kikuta, S., et al. (2010). The trehalose transporter 1 gene sequence is conserved in insects and encodes proteins with different kinetic properties involved in trehalose import into peripheral tissues. Insect Biochem. Mol. Biol. 40, 30-37. doi: 10.1016/j.ibmb.2009.12.006

Keeley, L. L., and Gäde, G. (2004). Adipokinetic and Hypertrehalosemic Neurohormones, in Encyclopedia of Entomology. Dordrecht: Springer.

Kelley, L. A., Mezulis, S., Yates, C. M., Wass, M. N., and Sternberg, M. J. (2015). The Phyre2 web portal for protein modeling, prediction and analysis. Nat. Protoc. 10, 845-858. doi: 10.1038/nprot.2015.053

Kh, S. D., and Keshan, B. (2019). Mobilization of fat body glycogen and haemolymph trehalose under nutritional stress in Bombyx mori larvae in relation to their physiological age and the duration of food deprivation. Biologia 74, 649-660. doi: 10.2478/s11756-019-00196-0

Kikawada, T., Saito, A., Kanamori, Y., Nakahara, Y., Iwata, K., Tanaka, D., et al. (2007). Trehalose transporter 1, a facilitated and high-capacity trehalose transporter, allows exogenous trehalose uptake into cells. Proc. Natl. Acad. Sci. U.S.A. 104, 11585-11590. doi: 10.1073/pnas.0702538104

Kikuta, S., Hagiwara-Komoda, Y., Noda, H., and Kikawada, T. (2012). A novel member of the trehalose transporter family functions as an $\mathrm{h}(+)$-dependent trehalose transporter in the reabsorption of trehalose in malpighian tubules. Front. Physiol. 3:290. doi: 10.3389/fphys.2012.00290

Kim, Y., and Hong, Y. (2015). Regulation of hemolymph trehalose level by an insulin-like peptide through diel feeding rhythm of the beet armyworm, Spodoptera exigua. Peptides 68, 91-98. doi: 10.1016/j.peptides.2015.02.003

Kumar, S., Stecher, G., Li, M., Knyaz, C., and Tamura, K. (2018). MEGA X: molecular evolutionary genetics analysis across computing platforms. Mol. Biol. Evol. 35, 1547-1549. doi: 10.1093/molbev/msy096

Lenaerts, C., Monjon, E., Van Lommel, J., Verbakel, L., and Vanden Broeck, J. (2019). Peptides in insect oogenesis. Curr. Opin. Insect Sci. 31, 58-64. doi: 10.1016/j.cois.2018.08.007

Leyria, J., Fruttero, L. L., Aguirre, S. A., and Canavoso, L. E. (2014). Ovarian nutritional resources during the reproductive cycle of the hematophagous Dipetalogaster maxima (Hemiptera: Reduviidae): focus on lipid metabolism. Arch. Insect Biochem. Physiol. 87, 148-163. doi: 10.1002/arch.211 86

Leyria, J., Orchard, I., and Lange, A. B. (2020a). Transcriptomic analysis of regulatory pathways involved in female reproductive physiology of Rhodnius prolixus under different nutritional states. Sci. Rep. 10:11431. doi: 10.1038/ s41598-020-67932-4

Leyria, J., Orchard, I., and Lange, A. B. (2020b). What happens after a blood meal? A transcriptome analysis of the main tissues involved in egg production in Rhodnius prolixus, an insect vector of Chagas disease. PLoS Negl. Trop. Dis. 14:e0008516. doi: 10.1371/journal.pntd.0008516

Li, J.-X., Cao, Z., Guo, Z., Tian, Z., Liu, W., Zhu, F., et al. (2020). Molecular characterization and functional analysis of two trehalose transporter genes in the cabbage beetle, Colaphellus bowringi. J. Asia Pac. Entomol. 23, 627-633. doi: 10.1016/j.aspen.2020.05.011

Li, L., Stoeckert, C. J. Jr., and Roos, D. S. (2003). OrthoMCL: identification of ortholog groups for eukaryotic genomes. Genome Res. 13, 2178-2189. doi: 10.1101/gr.1224503

Lindemans, M., Liu, F., Janssen, T., Husson, S. T., Mertens, I., Gäde, G., et al. (2009). Adipokinetic hormone signaling through the gonadotropin-releasing hormone receptor modulates egg-laying in Caenorhabditis elegans. Proc. Natl. Acad. Sci. U.S.A. 106, 1642-1647. doi: 10.1073/pnas.0809881106 
Liu, X., Zou, Z., Zhang, C., Liu, X., Wang, J., Xin, T., et al. (2020). Knockdown of the trehalose-6-phosphate synthase gene using rna interference inhibits synthesis of trehalose and increases lethality rate in asian citrus psyllid, Diaphorina citri (Hemiptera: Psyllidae). Insects 11:E605. doi: 10.3390/insects1109 0605

Livak, K. J., and Schmittgen, T. D. (2011). Analysis of relative gene expression data using real-time quantitative PCR and the 2(-Delta Delta C(T)) method. Methods 25, 402-408. doi: 10.1006/meth.2001.1262

Lorenz, M. W., and Anand, A. N. (2004). Changes in the biochemical composition of fat body stores during adult development of female crickets, Gryllus bimaculatus. Arch. Insect Biochem. Physiol. 56, 110-119. doi: 10.1002/arch. 20002

Lorenz, M. W., and Gäde, G. (2009). Hormonal regulation of energy metabolism in insects as a driving force for performance. Integr. Comp. Biol. 49, 380-392. doi: 10.1093/icb/icp019

Lu, K., Wang, Y., Chen, X., Zhang, X., Li, W., Cheng, Y., et al. (2019). Adipokinetic hormone receptor mediates trehalose homeostasis to promote vitellogenin uptake by oocytes in Nilaparvata lugens. Front. Physiol. 9:1904. doi: 10.3389/ fphys.2018.01904

Majerowicz, D., Alves-Bezerra, M., Logullo, R., Fonseca-de-Souza, A. L., MeyerFernandes, J. R., Braz, G. R., et al. (2011). Looking for reference genes for real-time quantitative PCR experiments in Rhodnius prolixus (Hemiptera: Reduviidae). Insect Mol. Biol. 20, 713-722. doi: 10.1111/j.1365-2583.2011. 01101.x

Marco, H. G., Simek, P., Clark, K. D., and Gäde, G. (2013). Novel adipokinetic hormones in the kissing bugs Rhodnius prolixus, Triatoma infestans, Dipetalogaster maxima and Panstrongylus megistus. Peptides 41, 21-30. doi: 10.1016/j.peptides.2012.09.032

Mariano, A. C., Santos, R., Gonzalez, M. S., Feder, D., Machado, E. A., Pascarelli, B., et al. (2009). Synthesis and mobilization of glycogen and trehalose in adult male Rhodnius prolixus. Arch. Insect Biochem. Physiol. 72, 1-15. doi: 10.1002/ arch.20319

Mesquita, R. D., Vionette-Amaral, R. J., Lowenberger, C., Rivera-Pomar, R., Monteiro, F. A., Minx, P., et al. (2015). Genome of Rhodnius prolixus, an insect vector of Chagas disease, reveals unique adaptations to hematophagy and parasite infection. Proc. Natl. Acad. Sci. U.S.A. 112, 14936-14941. doi: $10.1073 /$ pnas. 1506226112

Meyer, H., Vitavska, O., and Wieczorek, H. (2011). Identification of an animal sucrose transporter. J. Cell Sci. 124, 1984-1991. doi: 10.1242/jcs.082024

Michitsch, J., and Steele, J. E. (2008). Carbohydrate and lipid metabolism in cockroach (Periplaneta americana) fat body are both activated by low and similar concentrations of Peram-AKH II. Peptides 29, 226-234. doi: 10.1016/ j.peptides.2007.08.031

Millen, B. H., and Beckel, W. E. (1970). The effects of nutrient and non-nutrient diets on the fat body cell nuclei and nucleoli of starved nymphs of Rhodnius prolixus (Hemiptera). Can. J. Zool. 48, 489-493. doi: 10.1139/z70-083

Moriwaki, N., Matsushita, K., Nishina, M., and Kono, Y. (2003). High concentrations of trehalose in aphid hemolymph. Appl. Entomol. Zool. 38, 241-248. doi: 10.1303/aez.2003.241

Oda, Y., Uejima, M., Iwami, M., and Sakurai, S. (2000). Role of ecdysteroids in the dynamics of insect haemolymph sugar. Zool. Sci. 17, 785-789. doi: 10.2108/zsj. 17.785

Park, Y., and Kim, Y. (2017). Identification of a hypertrehalosemic factor in Spodoptera exigua. Arch. Insect Biochem. Physiol. 95:e21386. doi: 10.1002/arch. 21386

Parrou, J. L., and François, J. (1997). A simplified procedure for a rapid and reliable assay of both glycogen and trehalose in whole yeast cells. Anal. Biochem. 248, 186-188. doi: 10.1006/abio.1997.2138

Passier, P. C., Vullings, H. G., Diederenm, J. H., and Van der Horst, D. J. (1997). Trehalose inhibits the release of adipokinetic hormones from the corpus cardiacum in the African migratory locust, Locusta migratoria, at the level of the adipokinetic cells. J. Endocrinol. 153, 299-305. doi: 10.1677/joe.0.15302 99

Patel, H., Orchard, I., Veenstra, J. A., and Lange, A. B. (2014). Reprint of "The distribution and physiological effects of three evolutionarily and sequencerelated neuropeptides in Rhodnius prolixus: adipokinetic hormone, corazonin and adipokinetic hormone/corazonin-related peptide". Gen. Comp. Endocrinol. 203, 307-314. doi: 10.1016/j.ygcen.2014.07.001
Price, D. R., Tibbles, K., Shigenobu, S., Smertenko, A., Russell, C. W., Douglas, A. E., et al. (2010). Sugar transporters of the major facilitator superfamily in aphids; from gene prediction to functional characterization. Insect Mol. Biol. 2, 97-112. doi: 10.1111/j.1365-2583.2009.00918.x

Raikhel, A. S., and Dhadialla, T. S. (1992). Accumulation of yolk proteins in insect oocytes. Annu. Rev. Entomol. 37, 217-251. doi: 10.1146/annurev.en.37.010192. 001245

Reynolds, C. R., Islam, S. A., and Sternberg, M. J. E. (2018). EzMol: a web server wizard for the rapid visualization and image production of protein and nucleic acid structures. J. Mol. Biol. 430, 2244-2248. doi: 10.1016/j.jmb.2018.01.013

Roy, S., Saha, T. T., Zou, Z., and Raikhel, A. S. (2018). Regulatory pathways controlling female insect reproduction. Annu. Rev. Entomol. 63, 489-511. doi: 10.1146/annurev-ento-020117-043258

Santos, R., Alves-Bezerra, M., Rosas-Oliveira, R., Majerowicz, D., MeyerFernandes, J. R., and Gondim, K. C. (2012). Gene identification and enzymatic properties of a membrane-bound trehalase from the ovary of Rhodnius prolixus. Arch. Insect Biochem. Physiol. 81, 199-213. doi: 10.1002/arch.21043

Santos, R., Mariano, A. C., Rosas-Oliveira, R., Pascarelli, B., Machado, E. A., MeyerFernandes, J. R., et al. (2008). Carbohydrate accumulation and utilization by oocytes of Rhodnius prolixus. Arch. Insect Biochem. Physiol. 67, 55-62. doi: 10.1002/arch.20217

Satake, S., Masumura, M., Ishizaki, H., Nagata, H., Suzuki, A., and Mizoguchi, A. (1997). An insulin-related peptide of insects reduces the major storage carbohydrates in the silkworm Bombyx mori. Comp. Biochem. Physiol. B 118, 349-357. doi: 10.1016/s0305-0491(97)00166-1

Shukla, E., Thorat, L. J., Nath, B. B., and Gaikwad, S. M. (2015). Insect trehalase: physiological signi?cance and potential applications. Glycobiology 25, 357-367. doi: $10.1093 /$ glycob/cwu125

Sigrist, C. J. A., de Castro, E., Cerutti, L., Cuche, B. A., Hulo, N., Bridge, A., et al. (2013). New and continuing developments at PROSITE. Nucleic. Acids Res. 41, D344-D347. doi: 10.1093/nar/gks1067

Singtripop, T., Oda, Y., Wanichacheewa, S., and Sakurai, S. (2002). Sensitivities to juvenile hormone and ecdysteroid in the diapause larvae of Omphisa fuscidentalis based on the hemolymph trehalose dynamics index. J. Insect Physiol. 48, 817-824. doi: 10.1016/s0022-1910(02)00104-x

Steele, J. E. (1961). Occurence of a hyperglycaemic factor in the corpus cardiacum of an insect. Nature 18, 680-681. doi: 10.1038/192680a0

Steele, J. E. (1981). "The role of carbohydrate metabolism in physiological function," in Energy Metabolism in Insects, ed. R. G. H. Downer (Boston, MA: Springer), 101-133. doi: 10.1007/978-1-4615-9221-1_4

Su, Z. H., Ikedam, M., Sato, Y., Saito, H., Imai, K., Isobe, M., et al. (1994). Molecular characterization of ovary trehalase of the silkworm, Bombyx mori and its transcriptional activation by diapause hormone. Biochim. Biophys. Acta. 1218, 366-374. doi: 10.1016/0167-4781(94)90190-2

Süren-Castillo, S., Abrisqueta, M., and Maestro, J. L. (2014). FoxO is required for the activation of hypertrehalosemic hormone expression in cockroaches. Biochim. Biophys. Acta. 1840, 86-94. doi: 10.1016/j.bbagen.2013.08.015

Tang, B., Chen, J., Yao, Q., Pan, Z. Q., Xu, W. H., and Wang, S. G. (2010). Characterization of a trehalose-6-phosphate synthase gene from Spodoptera exigua, and its function identification through RNA interference. J. Insect Physiol. 56, 813-821. doi: 10.1016/j.jinsphys.2010. 02.009

Tang, B., Wang, S., Wang, S. G., Wang, H. J., Zhang, J. Y., and Cui, S. Y. (2018). Invertebrate trehalose-6-phosphate synthase gene: genetic architecture, biochemistry, physiological function, and potential applications. Front. Physiol. 9:30. doi: 10.3389/fphys.2018.00030

Tang, B., Xu, Q., Zou, Q., Fang, Q., Wang, S., and Ye, G. (2012). Sequencing and characterization of glycogen synthase and glycogen phosphorylase genes from Spodoptera exigua and analysis of their function in starvation and excessive sugar intake. Arch. Insect Biochem. Physiol. 80, 42-62. doi: 10.1002/arch.21027

Thompson, S. N. (2003). Trehalose - the insect "Blood" sugar. Adv. Insect Phys. 31, 205-285. doi: 10.1016/S0065-2806(03)31004-5

Toprak, U. (2020). The role of peptide hormones in insect lipid metabolism. Front. Physiol. 11:434. doi: 10.3389/fphys.2020.00434

Valle, D. (1993). Vitellogenesis in insects and other groups-a review. Mem. Inst. Oswaldo Cruz. 88, 1-26. doi: 10.1590/s0074-02761993000100005

Wang, S. S., Li, G. Y., Liu, Y. K., Luo, Y. J., Xu, C. D., Li, C., et al. (2020). Regulation of carbohydrate metabolism by trehalose-6-phosphate synthase 3 
in the brown Planthopper, Nilaparvata lugens. Front. Physiol. 11:575485. doi: 10.3389 /fphys.2020.575485

Wigglesworth, V. B. (1936). The function of the corpus allatum in the growth and reproduction of Rhodnius prolixus (Hemiptera). Q. J. Microsc. Sci. 79, 91-121.

World Health Organization [WHO] (2020). Chagas disease (American trypanosomiasis). Available online at: https://www.who.int/chagas/en/ (accessed March 30, 2020).

Wu, Q., and Brown, M. R. (2006). Signaling and function of insulin-like peptides in insects. Annu. Rev. Entomol. 51, 1-24. doi: 10.1146/annurev.ento.51.110104. 151011

Xie, Y., Zheng, Y., Li, H., Luo, X., He, Z., Cao, S., et al. (2016). GPS-Lipid: a robust tool for the prediction of multiple lipid modification sites. Sci. Rep. 6:28249. doi: $10.1038 /$ srep 28249

Xiong, K. C., Wang, J., Li, J. H., Deng, Y. Q., Pu, P., Fan, H., et al. (2019). RNA interference of a trehalose-6-phosphate synthase gene reveals its roles during larval-pupal metamorphosis in Bactrocera minax (Diptera: Tephritidae). J. Insect Physiol. 92, 84-92. doi: 10.1016/j.jinsphys.2016.07.003

Xu, J., Bao, B., Zhang, Z. F., Yi, Y. Z., and Xu, W. H. (2009). Identification of a novel gene encoding the trehalose phosphate synthase in the cotton bollworm, Helicoverpa armigera. Glycobiology 19, 250-257. doi: 10.1093/glycob/ cwn 127
Xu, J., Sheng, Z., and Palli, S. R. (2013). Juvenile hormone and insulin regulate trehalose homeostasis in the red flour beetle, Tribolium castaneum. PLoS Genet. 9:e1003535. doi: 10.1371/journal.pgen.1003535

Zandawala, M., Hamoudi, Z., Lange, A. B., and Orchard, I. (2015). Adipokinetic hormone signalling system in the Chagas disease vector, Rhodnius prolixus. Insect Mol. Biol. 24, 264-276. doi: 10.1111/imb.12157

Zheng, H., Chen, C., Liu, C., Song, Q., and Zhou, S. (2020). Rhythmic change of adipokinetic hormones diurnally regulates locust vitellogenesis and egg development. Insect Mol. Biol. 29, 283-292. doi: 10.1111/imb. 12633

Conflict of Interest: The authors declare that the research was conducted in the absence of any commercial or financial relationships that could be construed as a potential conflict of interest.

Copyright (๑ 2021 Leyria, El-Mawed, Orchard and Lange. This is an open-access article distributed under the terms of the Creative Commons Attribution License (CC BY). The use, distribution or reproduction in other forums is permitted, provided the original author(s) and the copyright owner(s) are credited and that the original publication in this journal is cited, in accordance with accepted academic practice. No use, distribution or reproduction is permitted which does not comply with these terms. 